\title{
The mean free path of electrons in metals*
}

\author{
E. H. Sondheimer $\dagger$ \\ Royal Society Mond Laboratory, Cambridge
}

\begin{tabular}{llr} 
& \multicolumn{1}{c}{ Contents } & PAGE \\
1. Introduction & 499 \\
2. Conduction in thin films and wires & 505 \\
3. Magnetic effects in thin conductors & 515 \\
4. The anomalous skin effect & 524 \\
Acknowledgement & 536 \\
References & 536
\end{tabular}

\section{Introduction}

1.1. The foundations of the modern electron theory of metals were laid at the beginning of the present century, when the existence of a gas of free electrons was postulated by Drude in order to explain the conducting properties of metals; the behaviour of the electrons was subsequently analysed by Lorentz by means of the statistical methods of the dynamical theory of gases. The chief success of the DrudeLorentz theory was the prediction of the Wiedemann-Frenz law connecting the electrical and thermal conductivities, but later developments revealed an increasing number of serious difficulties, outstanding among them the inability of the theory to explain why the conduction electrons do not contribute appreciably to the specific heat of a metal. This paradox was not resolved until the advent of quantum mechanics, when Pauli and Sommerfeld applied the Fermi-Dirac statistics to the free electrons in a metal, showing that in this way most of the contradictions could be reconciled.

The Drude-Lorentz-Sommerfeld theories are essentially formal in character. They involve as arbitrary parameters the number $n$ of free electrons per unit volume, which is assumed to be of the same order as the number of atoms per unit volume, and the mean free path $l$ of the electrons which is to be determined from a comparison of theory and experiment. So far as the electrical conductivity $\sigma_{0}$ is concerned, the results of the Sommerfeld theory are summarized by the formulae

$$
\begin{aligned}
n & =\frac{8 \pi}{3}\left(\frac{m \bar{v}}{h}\right)^{3}, \\
\sigma_{0} & =\frac{n \epsilon^{2} l}{m \bar{v}}
\end{aligned}
$$

* This article was the first to appear in Advances in Physics, originally published in volume 1, 1952. It had attracted 1030 citations by October 2001, and is ranked 5 in the index of articles attracting more than 100 citations.

$\dagger$ Fellow of Trinity College, Cambridge.

Advances in Physics ISSN 0001-8732 print/ISSN 1460-6976 online (C) 2001 Taylor \& Francis Ltd 
in which $h$ is Planck's constant, $-\epsilon$ is the charge and $m$ is the mass of an electron, and $\bar{v}$ is the velocity of an electron at the surface of the Fermi distribution. $\sigma_{0}$ therefore depends on $n, l$ and fundamental constants only. In order to obtain the correct order of magnitude for the conductivity and the correct temperature variation, it is necessary to assume that $l$ is of the order of several hundred interatomic distances at ordinary temperatures, and increases rapidly in a pure metal towards very low temperatures.

Such long free paths which vary with temperature are very difficult to explain on classical theory, but they can be understood on the basis of the recent developments in the theory of metals. These were initiated by Bloch and elaborated by a great many authors; they have been concerned with a detailed quantum-mechanical analysis of the motion of electrons in a crystal latice, and they have made it possible to give precise meanings to the two fundamental concepts of the 'number of free electrons' and the 'mean free path' and to obtain numerical estimates of these quantities in certain cases. The electrons in a metal are regarded as distributed over a number of energy bands, filling most of them completely. All the electrons are free to move through the lattice, but only those which are contained in incompletely filled energy bands can contribute to the resultant current and are to be regarded as free electrons for the purposes of conduction theory. The number of free electrons in a metal is of the same order as the number of atoms: the precise number, however, depends on the detailed configuration of the energy bands and need not be a simple multiple or submultiple of the number of atoms. The variation of $n$ with temperature is negligible, since at ordinary temperatures the free electrons form a highly degenerate Fermi-Dirac gas.

An electron can move freely through a perfect and rigid crystal lattice and there is no resistance. In a pure metal a finite free path is caused by the thermal vibrations of the lattice and is of the same order as the average wavelength of the sound waves in the metal, which is large compared with the interatomic distance and is increased by lowering the temperature. The free path does not increase indefinitely, however, as the temperature is lowered, and at very low temperatres it tends to a constant 'residual' value $l_{r}$ which is determined by static lattice imperfections such as the presence of impurity atoms, and which is of the order of the distance between the impurities.

The following theoretical formula may be deduced for the electrical conductivity of a metal, subject to many simplifying assumptions concerning the interaction between the electrons and the lattice vibrations (see, for example, Wilson 1936, Chapter VI):

$$
\frac{1}{\sigma_{0}}=\frac{m \bar{v}}{n \epsilon^{2} l_{r}}+\left(\frac{m}{2}\right)^{1 / 2} \frac{9 \pi h^{2} C^{2}}{8 n \Delta \epsilon^{2} M k \Theta \zeta^{3 / 2}}\left(\frac{T}{\Theta}\right)^{5} \int_{0}^{\theta / \mathrm{T}} \frac{z^{5} \mathrm{~d} z}{\left(e^{z}-1\right)\left(1-e^{-z}\right)}
$$

Here $k$ is Boltzmann's constant, $\zeta$ is the Fermi energy level $\left(\zeta=\frac{1}{2} m \bar{v}^{2}\right), \Theta$ is the Debye temperature, $M$ is the mass of an atom, $\Delta$ is the volume of the unit cell, and $C$ is a constant which determines the interaction between the electrons and the lattice. According to this formula the 'ideal' and 'residual' resistances are additive, and the ideal resistance is proportional to $T$ at high and to $T^{5}$ at very low temperatures. (For commercially pure metals the residual resistance can be neglected at ordinary temperatures.) The temperature variation of the resistance predicted by (2) is, generally speaking, in good agreement with observation, particularly for the 
monovalent metals. The absolute value of the free path at any temperature can be estimated by combining (1) and (2) and substituting reasonable values for the parameters. The chief uncertainty concerns the magnitude of the interaction constant $C$, estimates of which can be obtained by numerical integration if the wave functions of the conduction electrons are known; it is of the same order as the Fermi energy $\zeta$. The estimates of the free path are of the right order of magnitude, but precise numerical values cannot be obtained in this way.

1.2. An important conclusion to be drawn from the detailed quantum-mechanical theory is that the simple Sommerfeld treatment of the conduction phenomena remains correct within certain limits. In the Sommerfeld theory the electrons are regarded as perfectly free, their energy being proportional to the square of the velocity; this remains approximately true for electrons moving in a lattice, but the mass $m$ which appears in equations (1) and (2) must be regarded as an effective mass which is of the same order of magnitude as, but not necessarily equal to, the mass of a free electron. This model of 'quasi-free' electrons, which is implicit in the derivation of (2), applies most closely to the monovalent metals in which the conduction electrons are all contained in a single energy band; for these metals, moreover, the number of conduction electrons should be precisely one per atom. In multivalent metals, in which the electrons occupy more than one band, the model may still be used to give a semi-quantitative description of the simpler conduction phenomena, but quantities such as $n$ and $m$ must then be regarded as representing certain averages of the numbers of electrons and the effective masses of the electrons in the various bands, and the precise numerical values have no immediate physical significance. It would, of course, be possible to consider more complicated models, for example one in which the conduction electrons are contained in two overlapping energy bands. In the present state of the theory of metals, however, it is impossible to work out a theory which fully takes into account the electronic structure peculiar to any particular metal, and instead of introducing a large number of parameters of doubtful physical significance it is best to work with the simplest model which gives reasonable results. For more complicated conduction phenomena, however, particularly those which are associated with the presence of a magnetic field or with anisotropy effects, the free-electron model is entirely inadequate (it leads, for example, to a zero magneto-resistance effect), and even a qualitative theory can only be obtained by using a model in which the energy surfaces do not form a singlyconnected set of spheres.

In the Sommerfeld theory the free path is most conveniently introduced through the time of relaxation $\tau$, which is defined as follows. Let $\mathbf{v}=\left(v_{x}, v_{y}, v_{z}\right)$ be the velocity of an electron, and let $2(m / h)^{3} f(\mathbf{v}, \mathbf{r}) \mathrm{d} \mathbf{r} \mathrm{d} \mathbf{v}$ be the number of electrons in the volume element $\mathrm{d} \mathbf{r}=\mathrm{d} x \mathrm{~d} y \mathrm{~d} z$ which have their velocities in the range $\mathrm{d} \mathbf{v}=\mathrm{d} v_{x} \mathrm{~d} v_{y} \mathrm{~d} v_{z} ; f$ is the distribution function as usually defined in Fermi-Dirac statistics. Suppose that some non-equilibrium distribution function is set up by a system of external forces which are suddenly removed; the rate of approach to equilibrium under the influence of collisions alone is then supposed to be given by

$$
\left[\frac{\partial f}{\partial t}\right]_{\mathrm{coll}}=-\frac{f-f_{0}}{\tau}
$$


where $f_{0}$ is the equilibrium distribution function. The time of relaxation need not be a constant and may depend, for example, on the velocity; if $v$ is the mean velocity of those electrons to which $\tau$ refers, the corresponding free path $l$ is defined by $l=v \tau$. In general $[\partial f / \partial t]_{\text {coll }}$ takes the form of an integral operator which does not reduce to the simple form (3); it is then impossible to define a free path in any natural way. The detailed theory of the conduction mechanism in metals shows, however, that a free path does exist in the above sense under certain conditions for quasi-free conduction electrons; thus it can always be defined for scattering by randomly distributed impurity atoms, and it also exists for scattering by lattice vibrations if the temperature is above the Debye temperature. So far as the electrical conductivity is concerned, it is a reasonble approximation to assume that a free path can be defined for all temperatures, so that equation (1) holds and the temperature variation of the free path is the same as that of the electrical conductivity. An approximate theoretical expression for $l$ in terms of the atomic constants of the metal can be obtained by combining equations (1) and (2). It must be borne in mind, however, that the free path associated in this way with the electrical conductivity is not necessarily the same as the free path associated, for example, with the thermal conductivity.

1.3. With all the provisos mentioned, then, the simple Sommerfeld picture of the free electrons in a metal and their mean free path retains its validity and may be used to discuss the conduction phenomena. It is therefore an important problem to determine the various parameters of the theory by as many independent methods as possible; such estimates are of interest in themselves in providing information about the electronic structure of metals, and they serve as a valuable check on the consistency of the free-electron theory. Furthermore, since the calculation of the free path from fundamental principles is highly complicated and involves many drastic approximations, it is desirable to have methods by which $l$ may be estimated directly from observational data.

The simplest procedure (Mott and Jones 1936) is to compare equation (1) with the observed electrical conductivity, but this gives only $n^{2 / 3} l$ and $l$ cannot be obtained unless $n$ can be estimated independently. $\uparrow$ It is, however, possible to obtain $l$ directly by measuring the conductivity under conditions where the free path may be compared with some other characteristic length in the metal. Effects of this type have attracted much attention in the last few years, and it is with them that we shall henceforth be concerned.

The most obvious method is to use a thin film or wire and to arrange that the free path is comparable in magnitude with the thickness or diameter of the specimen; the artificial limitation of the free path by the boundaries of the specimen causes an increase in the resistivity above its value in the bulk metal, and this may be used to deduce the ratio of free path to thickness or diameter. This topic is reviewed in section 2 .

In section 3 we consider the more complicated effects which occur when a thin specimen is placed in a magnetic field. The ordinary bulk magneto-resistance effect depends in a complicated way on the binding of the electrons in the lattice and is zero

$\dagger$ It should be remarked that the value of $l$ defined by Mott and Jones and tabulated on p. 268 of their book is twice the free path defined here; the latter is the physically relevant quantity. 
for quasi-free electrons; in thin specimens, however, where boundary scattering of electrons is important, the alteration of the electron trajectories in a magnetic field in general leads to a non-zero change of resistance even if the electrons are regarded as free. These curious 'geometrical' effects, being classical in nature, are entirely different from the bulk effect and are essentially simpler to understand. The details of the phenomena vary with the shape of the specimen and with the relative configurations of specimen, current and magnetic field; their analysis in all cases involves a new quantity with the dimensions of length, namely the radius $r_{0}=m \bar{v} c / \epsilon H$ of a free-electron orbit in a magnetic field $H$. The experiments therefore give, in addition to the free path, a direct estimate of the momentum $m \bar{v}$ of the electrons at the surface of the Fermi distribution, and hence of the number of free electrons according to equation $(1 a)$. The method is, however, severely restricted in practice by the disturbing effect of the bulk magneto-resistance phenomenon.

In section 4, finally, we consider the so-called anomalous skin effect in metals. This is a more sophisticated side effect in which the free path is compared, not with the physical dimensions of the specimen, but with the distance to which a highfrequency electric field penetrates into the metal. Experiments on the high-frequency skin resistance of metals allow values of $l / \delta$ to be deduced, where $\delta$ is the classical skin penetration depth, and since $\delta$ depends only on the frequency and the d.c. electrical conductivity, the free path can again be obtained directly from experimental magnitudes.

1.4. These phenomena present interesting problems from both experimental and theoretical points of view. Let us consider the orders of magnitude of the various characteristic lengths. The free path in metals at room temperatures is of the order of $10^{-5} \mathrm{~cm}$ or less, but in a pure metal at liquid-helium temperatures the high values of the conductivity indicate that it may be as large as $10^{-2} \mathrm{~cm}$. It is clear, therefore, that extremely thin films would be required in experiments on the size effect at normal temperatures; such films are difficult to prepare and usually show subsidiary resistive effects, often time dependent, which tend to obscure the pure geometrical limitation of the free path with which we are alone concerned. In order to obtain results which are free from ambiguity, it is therefore essential to carry out the experiments at very low temperatures where relatively large specimens (of thickness $\sim 10^{-3} \mathrm{~cm}$ ) may be used. The necessity for using specimens of this order of thickness becomes even greater when we consider the effects which take place in a magnetic field. These effects show up when the orbit radius $r_{0}$ is comparable with the thickness of the specimen. Since $\bar{v}$ is of the order of $10^{8} \mathrm{~cm} / \mathrm{sec}$, a magnetic field of reasonable magnitude (several kilogauss) corresponds to $r_{0} \sim 10^{-3} \mathrm{~cm}$; and since $r_{0}$ is inversely proportional to $H$, impracticably large magnetic fields would be required in the case of films much thinner than this. Finally, in the anomalous skin effect $l$ must be large compared with $\delta$; the ratio $l / \delta$ is proportional to $l^{3 / 2}$ and to the square root of the frequency, so that both very low temperatures and high frequencies are required. For a pure metal at liquid-helium temperatures and for microwave frequencies, $l / \delta$ is of the order of 100 .

One may say, therefore, that the size effects are essentially low-temperature phenomena. Since the free path at low temperatures varies from specimen to specimen and is not a characteristic property of the metal, it is usual to measure (or estimate in some way) the value of the bulk conductivity $\sigma_{0}$ which corresponds to the free path $l$, and to express the results of the experiments in terms of the ratio $\sigma_{0} / l$. 
This ratio determines directly the number of conduction electrons per unit volume, since, according to equations $(1 a)$ and $(1 b)$,

$$
\frac{\sigma_{0}}{l}=\frac{n \epsilon^{2}}{m \bar{v}}=\left(\frac{8 \pi}{3}\right)^{1 / 3} \frac{\epsilon^{2} n^{2 / 3}}{h}=7.1 \times 10^{7} n^{2 / 3} .
$$

Note that this relation involves only the assumption that the electrons are quasi-free, but not that their effective mass is necessarily equal to the mass of a free electron.

1.5. Many approximate theoretical treatments have been given of the various free-path phenomena, but we shall not refer to these (except where they are particularly relevant to the discussion), as they have been superseded by the strict statistical analysis based on the Boltzmann equation for the distribution function of the conduction electrons. $t$ This equation is formed by equating the rate of change in $f$ due to external fields to the rate of change due to the collision mechanism, which is assumed to be given by equation (3). In the presence of an electric field $\mathbf{E}$ and a magnetic field $\mathbf{H}$, the Boltzmann equation for quasi-free electrons takes the form

$$
-\frac{\epsilon}{m}\left(\mathbf{E}+\frac{1}{c} \mathbf{v} \times \mathbf{H}\right) \cdot \operatorname{grad}_{\mathbf{v}} f+\mathbf{v} \cdot \operatorname{grad}_{\mathbf{r}} f=-\frac{f-f_{0}}{\tau},
$$

which is purely classical except that the mass $m$ is to be regarded as an effective mass, while the equilibrium distribution function is the Fermi-Dirac function

$$
f_{0}(E)=\frac{1}{e^{(E-\zeta) / k T}+1} .
$$

The time of relaxation $\tau$ is supposed to depend on the absolute value of $\mathbf{v}$ only.

$f$ is a function of the velocity vector $\mathbf{v}$ and the space vector $\mathbf{r}$, and the analysis of the size effects depends essentially on the appearance in equation (5) of the term involving the space derivatives of $f$, which is required in the bulk metal only if temperature gradients are present. This term takes into account the non-uniform distribution in space of the conduction electrons which is characteristic of the sizeeffect phenomena. A general feature of the analysis is that boundary conditions have to be imposed on the solutions of the Boltzmann equation; these are determined in each case by the nature of the scattering at the physical boundaries of the specimen.

In treating the various problems in this way we may apply the results directly to the alkali metals, in which the electrons are most nearly free, and with somewhat lesser confidence to the noble metals; for more complex metals the model will provide only a qualitative picture, but should give the orders of magnitude correctly. In the presence of a magnetic field, corrections of uncertain amount must be applied to the classical formulae to allow both for the bulk magneto-resistance effect and, possibly, for the effect of the quantization of the electron orbits in a magnetic field. These refinements are difficult to take into account in any satisfactory way; the most

$\dagger$ Gaussian units are used throughout the present article.

$\$$ The use of the Boltzmann equation in this connection appears to have been first suggested by Peierls.

$\S E$ here denotes the energy of an electron, but elsewhere in this article, $E$ stands for electric field strength. 
reasonable procedure, therefore, is to neglect them entirely and to restrict the experiments to metals in which they are of least importance (see section 3.1).

The main problem, then, is to solve equation (5) for the various cases of interest, and to use the solution to calculate the current density $\mathbf{J}$ by means of the usual formula

$$
\mathbf{J}=-2 \epsilon\left(\frac{m}{h}\right)^{3} \int \mathbf{v} f \mathrm{~d} \mathbf{v} .
$$

1.6. Objections have been raised from time to time against the whole basis of the theory of metallic conductivity: thus the uncertainty principle has been used to argue that the uncertainty in the energy of a single electron, which is determined by the magnitude of the time of relaxation, may be so large in some cases as to render meaningless any description of the situation by means of a distribution function which obeys the classical Boltzmann equation (for a detailed discussion of this somewhat oversimplified statement compare, for example, Peierls 1934). These objections have never been satisfactorily met, and it must be admitted that there is no entirely rigorous quantum theory of conductivity. So far as the effects considered here are concerned, however, it may be argued that the free path appears as a parameter which need not itself be determined by the theory and which determines the phenomena in an essentially classical way; since the physical concepts involved are classical, it is not perhaps surprising that the effects can be explained by a theory which employs essentially classical methods. In any case, the great success of the theory in explaining the experimental facts strongly encourages belief in the basic validity of its assumptions.

\section{Conduction in thin films and wires}

\subsection{Thin films}

The strict analysis of the conductivity of a thin metallic film was given by Fuchs (1938). The method is typical of all strict calculations on the various size effects and will therefore be presented in detail.

Consider a metal film of thickness $a$ and suppose that the $z$-axis is perpendicular to the plane of the film, the surfaces of the film being the planes $z=0$ and $z=a$. The problem is essentially a one-dimensional one, and the distribution function of the electrons may be written in the form

$$
f=f_{0}+f_{1}(\mathbf{v}, z)
$$

where the function $f_{1}$ which has to be determined depends on the space variables only through $z$. The electric field $E$ is supposed to be in the $x$-direction, and, if we neglect the product of $E$ with $f_{1}$ (which is permissible so long as we are not interested in deviations from Ohm's law), the Boltzmann equation reduces to

$$
\frac{\partial f_{1}}{\partial z}+\frac{f_{1}}{\tau v_{z}}=\frac{\epsilon E}{m v_{z}} \frac{\partial f_{0}}{\partial v_{x}} .
$$

The general solution is easily written down, and is

$$
f_{1}(\mathbf{v}, z)=\frac{\epsilon \tau E}{m} \frac{\partial f_{0}}{\partial v_{x}}\left\{1+F(\mathbf{v}) \exp \left(-\frac{z}{\tau v_{z}}\right)\right\},
$$

where $F(\mathbf{v})$ is an arbitrary function of $\mathbf{v}$. 
2.11. To determine $F(\mathbf{v})$ we have to introduce the boundary conditions at the surfaces of the film. The simplest assumption is to suppose that every free path is terminated by collision at the surface, so that the scattering is entirely diffuse. The distribution function of the electrons leaving each surface must then be independent of direction; equation (10) shows that this can only be satisfied if we choose $F(\mathbf{v})$ so that $f_{1}(\mathbf{v}, 0)=0$ for all $\mathbf{v}$ such that $v_{z}>0$ (that is, for electrons moving away from the surface $z=0)$, and $f_{1}(\mathbf{v}, a)=0$ for all $\mathbf{v}$ such that $v_{z}<0$. There are therefore two distribution functions, $f_{1}^{+}$for electrons with $v_{z}>0$ and $f_{1}^{-}$for electrons with $v_{z}<0$; they are

$$
\left.\begin{array}{ll}
f_{1}^{+}(\mathbf{v}, z)=\frac{\epsilon \tau E}{m} \frac{\partial f_{0}}{\partial v_{x}}\left\{1-\exp \left(-\frac{z}{\tau v_{z}}\right)\right\} & \left(v_{z}>0\right), \\
f_{1}^{-}(\mathbf{v}, z)=\frac{\epsilon \tau E}{m} \frac{\partial f_{0}}{\partial v_{x}}\left\{1-\exp \left(\frac{a-z}{\tau v_{z}}\right)\right\} & \left(v_{z}<0\right) .
\end{array}\right\}
$$

2.1.1. We can now proceed with the calculation of the current density $J(z)$. Combining (7), (8), (11), introducing polar coordinates $(v, \theta, \phi)$ in the $\mathbf{v}$-space (with $\left.v_{z}=v \cos \theta\right)$, and remembering that $f_{0}$ depends on $v=|\mathbf{v}|$ only, we obtain

$$
\begin{aligned}
J(z)= & -\frac{2 \epsilon^{2} m^{2} E}{h^{3}} \int_{0}^{\infty} \mathrm{d} v \int_{0}^{2 \pi} \mathrm{d} \phi \tau v^{3} \cos ^{2} \phi \frac{\partial f_{0}}{\partial v} \\
& \times\left[\int_{0}^{\pi / 2} \sin ^{3} \theta\left\{1-\exp \left(-\frac{z}{\tau v \cos \theta}\right)\right\} \mathrm{d} \theta\right. \\
& \left.+\int_{\pi / 2}^{\pi} \sin ^{3} \theta\left\{1-\exp \left(\frac{a-z}{\tau v \cos \theta}\right)\right\} \mathrm{d} \theta\right] .
\end{aligned}
$$

The integration over $\phi$ is trivial, and the integration over $v$ is carried out by means of the formula

$$
-\int_{0}^{\infty} \psi(v) \frac{\partial f_{0}}{\partial v} \mathrm{~d} v=\psi(\bar{v})
$$

which holds for a degenerate electron gas. This gives, after some rearrangement,

$$
J(z)=\frac{4 \pi \epsilon^{2} m^{2} \tau \bar{v}^{3}}{h^{3}} E \int_{0}^{\pi / 2} \sin ^{3} \theta\left\{1-\exp \left(-\frac{a}{2 l \cos \theta}\right) \cosh \left(\frac{a-2 z}{2 l \cos \theta}\right)\right\} \mathrm{d} \theta
$$

where $l=\tau \bar{v}$ is the free path of the electrons at the surface of the Fermi distribution.

Equation (14) gives the current distribution across the thickness of the film. For comparison with experiment, however, we require the apparent overall conductivity of the film, and we must therefore average the current density over all values of $z$ from 0 to $a$. Carrying out the integration over $z$, we obtain for the effective conductivity

$$
\sigma=\frac{1}{E a} \int_{0}^{a} J(z) \mathrm{dz}=\sigma_{0}\left[1-\frac{3 l}{2 a} \int_{0}^{\pi / 2} \sin ^{3} \theta \cos \theta\left\{1-\exp \left(-\frac{a}{l \cos \theta}\right)\right\} \mathrm{d} \theta\right],
$$

$\sigma_{0}$ being the conductivity of the bulk metal, which is given by equation $(1 b)$. The ratio of the resistivity $1 / \sigma$ of the film to that of the bulk metal $1 / \sigma_{0}$ is most 
conveniently written in the form, obtained from (15) by means of a simple substitution,

$$
\frac{\sigma_{0}}{\sigma}=\frac{\Phi(\kappa)}{\kappa}
$$

where $\kappa=a / l$ and where

$$
\frac{1}{\Phi(\kappa)}=\frac{1}{\kappa}-\frac{3}{8 \kappa^{2}}+\frac{3}{2 \kappa^{2}} \int_{1}^{\infty}\left(\frac{1}{t^{3}}-\frac{1}{t^{5}}\right) e^{-\kappa t} \mathrm{~d} t .
$$

Repeated integration by parts gives the alternative expression

$$
\frac{1}{\Phi(\kappa)}=\frac{1}{\kappa}-\frac{3}{4}\left(1-\frac{1}{12} \kappa^{2}\right) E i(-\kappa)-\frac{3}{8 \kappa^{2}}\left(1-e^{-\kappa}\right)-\left(\frac{5}{8 \kappa}+\frac{1}{16}-\frac{\kappa}{16}\right) e^{-\kappa}
$$

where

$$
-E i(-u)=\int_{u}^{\infty} \frac{e^{-t}}{t} \mathrm{~d} t
$$

which is a tabulated integral; this form of the result is particularly convenient for computation.

The limiting form for large $\kappa$ (thick films) is

$$
\frac{\sigma_{0}}{\sigma}=1+\frac{3}{8 \kappa} \quad(\kappa \gg 1)
$$

and for very thin films we have

$$
\frac{\sigma_{0}}{\sigma}=\frac{4}{3 \kappa \log (1 / \kappa)} \quad(\kappa \ll 1) .
$$

These formulae are discussed further in section 2.3.

2.1.2. A somewhat more general theory, which does not assume that the scattering at the surface of the film is entirely diffuse, can be obtained as follows. We assume that a fraction $p$ of the electrons is scattered elastically at the surface with reversal of the velocity component $v_{z}$, while the rest are scattered diffusely with complete loss of their drift velocity. $p$ is supposed to be a constant independent of the direction of motion of the electrons. This is of course a highly artificial model, which in effect merely interpolates between the extreme cases of perfectly diffuse reflection, considered above, and perfectly specular reflection, for which the conductivity is unaltered. However, in the absence of any detailed theory of the nature of the surface scattering mechanism it is best to work with the simplest possible assumptions.

The distribution function of the electrons leaving the surface $z=0$ is now given by

$$
f_{0}+f_{1}^{+}\left(v_{z}, z=0\right)=p\left\{f_{0}+f_{1}^{-}\left(-v_{z}, z=0\right)\right\}+(1-p) f_{0},
$$

and similarly, at $z=a$,

$$
f_{0}+f_{1}^{-}\left(v_{z}, z=a\right)=p\left\{f_{0}+f_{1}^{+}\left(-v_{z}, z=a\right)\right\}+(1-p) f_{0} .
$$


Table 1. The resistivity of thin metallic films divided by the resistivity of the bulk metal.

\begin{tabular}{ccc}
\hline & \multicolumn{2}{c}{$\sigma_{0} / \sigma$} \\
\cline { 2 - 3 } (thickness/free path) & $p=0$ & $p=\frac{1}{2}$ \\
\hline 0.001 & 182 & 73.5 \\
0.002 & 100.4 & 41.5 \\
0.005 & 46.6 & 20.0 \\
0.01 & 26.5 & 11.8 \\
0.02 & 15.3 & 7.1 \\
0.05 & 7.69 & 3.87 \\
0.1 & 4.72 & 2.62 \\
0.2 & 3.00 & 1.91 \\
0.5 & 1.90 & 1.402 \\
1 & 1.462 & 1.206 \\
2 & 1.221 & 1.102 \\
5 & 1.081 & 1.039 \\
10 & 1.0390 & 1.0191 \\
20 & 1.0191 & 1.0095 \\
50 & 1.0076 & 1.0038 \\
100 & 1.0038 & 1.0019 \\
\hline
\end{tabular}

These equations are sufficient to determine $F(\mathbf{v})$, and instead of (11) we obtain for the distribution function

$$
\left.\begin{array}{ll}
f_{1}^{+}(\mathbf{v}, z)=\frac{\epsilon \tau E}{m} \frac{\partial f_{0}}{\partial v_{x}}\left\{1-\frac{1-p}{1-p \exp \left(-a / \tau v_{z}\right)} \exp \left(-\frac{z}{\tau v_{z}}\right)\right\} & \left(v_{z}>0\right), \\
f_{1}^{-}(\mathbf{v}, z)=\frac{\epsilon \tau E}{m} \frac{\partial f_{0}}{\partial v_{x}}\left\{1-\frac{1-p}{1-p \exp \left(a / \tau v_{z}\right)} \exp \left(\frac{a-z}{\tau v_{z}}\right)\right\} & \left(v_{z}<0\right) .
\end{array}\right\}
$$

The current density is calculated as before, and the result is that the function $\Phi(\kappa)$ defined by (17) must be replaced by $\Phi_{p}(\kappa)$, where

$$
\frac{1}{\Phi_{p}(\kappa)}=\frac{1}{\kappa}-\frac{3}{2 \kappa^{2}}(1-p) \int_{1}^{\infty}\left(\frac{1}{t^{3}}-\frac{1}{t^{5}}\right) \frac{1-e^{-\kappa t}}{1-p e^{-\kappa t}} \mathrm{~d} t .
$$

This reduces to (17) when $p=0$, and to the bulk-metal value $1 / \kappa$ when $p=1$. It is, of course, again possible to write down alternative forms of (25) more convenient for numerical work; compare, for example, equation (31). Instead of (20) and (21), we have

$$
\frac{\sigma_{0}}{\sigma}=1+\frac{3}{8 \kappa}(1-p) \quad(\kappa \gg 1)
$$

and

$$
\frac{\sigma_{0}}{\sigma}=\frac{4}{3} \frac{1-p}{1+p} \frac{1}{\kappa \log (1 / \kappa)} \quad(\kappa \ll 1) .
$$

Values of $\sigma_{0} / \sigma$ for $p=0$ and $\frac{1}{2}$ are shown in Table 1 . 


\subsection{Thin wires}

The corresponding analysis for the case of wires has been carried out by MacDonald and Sarginson (1950), who considered a wire of square cross-section, and by Dingle (1950) for the more important but more difficult case of a wire of circular cross-section. The method is essentially the same as that for a film, but the details of the analysis, for which the reader is referred to the original papers, are more complicated because the problems are now two-dimensional and the Boltzmann equation takes the form of a partial differential equation in the space variables. The result for a cylindrical wire of diameter $a$ is, $\dagger$ for $p=0$,

$$
\frac{\sigma_{0}}{\sigma}=\frac{\Psi(\kappa)}{\kappa}
$$

where $\kappa=a / l$,

$$
\frac{1}{\Psi(\kappa)}=\frac{1}{\kappa}-\frac{12}{\pi \kappa} \int_{0}^{1}\left(1-t^{2}\right)^{1 / 2} S_{4}(\kappa t) \mathrm{d} t,
$$

and

$$
S_{n}(u)=\int_{1}^{\infty} e^{-u t}\left(t^{2}-1\right)^{1 / 2} t^{-n} \mathrm{~d} t .
$$

The result for $p \neq 0$ may be derived from that for $p=0$ by means of the simple relation

$$
\left(\frac{\sigma}{\sigma_{0}}\right)_{\kappa, p}=(1-p)^{2} \sum_{n=1}^{\infty} n p^{n-1}\left(\frac{\sigma}{\sigma_{0}}\right)_{n \kappa, p=0} ;
$$

this holds incidentally also for the case of a thin film, as may be shown by expanding (25) in ascending powers of $p$ and comparing with (17).

For very thick and very thin wires the results reduce to

$$
\frac{\sigma_{0}}{\sigma}=1+\frac{3}{4 \kappa}(1-p) \quad(\kappa \gg 1)
$$

and

$$
\frac{\sigma_{0}}{\sigma}=\frac{1-p}{1+p} \frac{1}{\kappa} \quad(\kappa \ll 1) .
$$

(32) holds also for a square wire ( $\kappa$ being $a / l$ as before where $a$ is now the side of the wire), but (33) must be replaced by

$$
\frac{\sigma_{0}}{\sigma}=\frac{1-p}{1+p} \frac{0.897}{\kappa} \quad(\kappa \ll 1) .
$$

Numerical values of $\sigma_{0} / \sigma$ for a cylindrical wire are shown in Table 2.

\subsection{Discussion of the formulae}

It will be noted that, although the general theory is complicated, relatively simple formulae are obtained for specimens which are either thick or thin compared with the free path. The results for these limiting cases in fact possess a simple interpretation. 
Table 2. The resistivity of thin wires divided by the resistivity of the bulk metal.

\begin{tabular}{ccc}
\hline & \multicolumn{2}{c}{$\sigma_{0} / \sigma$} \\
\cline { 2 - 3 } (thickness/free path) & $p=0$ & $p=\frac{1}{2}$ \\
\hline 0.001 & 1000 & 337 \\
0.002 & 503 & 170 \\
0.005 & 202 & 69.3 \\
0.01 & 102 & 35.7 \\
0.02 & 52.1 & 18.7 \\
0.05 & 21.6 & 8.31 \\
0.1 & 11.45 & 4.88 \\
0.2 & 6.33 & 3.02 \\
0.5 & 3.14 & 1.84 \\
1 & 2.04 & 1.422 \\
2 & 1.475 & 1.208 \\
5 & 1.172 & 1.080 \\
10 & 1.081 & 1.038 \\
20 & 1.0390 & 1.0191 \\
50 & 1.0152 & 1.0076 \\
100 & 1.0076 & 1.0038 \\
\hline
\end{tabular}

2.3.1. The results given above for thick specimens are particular cases of a formula applicable to wires with cross-sections of arbitrary shape, the general form of which follows from simple dimensional considerations (Dingle 1950). When the thickness is large compared with the free path, the deviation in the current density from that which would exist in the bulk metal for the same applied field is appreciable only in a region close to the surface of the specimen. The deviation in the current is proportional to the perimeter $P$ of the cross-section, whilst the mean current is proportional to the cross-sectional area $S$. Hence

$$
\frac{\sigma_{0}}{\sigma}=1+\frac{P}{S} \times \text { constant }=1+C l P / S,
$$

where $C$ is a dimensionless constant independent of the shape of the specimen. For a thin film of thickness $a, P / S=2 / a$, and comparison with (26) shows that $C=3(1-p) / 16$. Therefore, for a wire of arbitrary cross-section,

$$
\frac{\sigma_{0}}{\sigma}=1+\frac{3}{16}(1-p) \frac{l P}{S} .
$$

In the notation of section $2.2, P / S=4 / a$ for both square and circular wires, and we regain (32).

2.3.2. We may define an 'effective' free path $l_{\text {eff }}$ for conduction in thin specimens by writing the effective conductivity in the standard form $\sigma=n \epsilon^{2} l_{\text {eff }} / m \bar{v}$. For wires of diameter small compared with the free path, we see from equations (33) and (34) that $l_{\text {eff }}=a$, apart from a constant of the order of unity. This result is intuitively obvious; for a very thin film, however, we find that $l_{\text {eff }} \approx a \log (l / a)$, so that $l_{\text {eff }}$ tends to infinity with $l$. The qualitative difference between the behaviour of thin films and of wires may at first sight seem surprising; its origin 
becomes more apparent if we consider a simple qualitative treatment of conduction in thin films due to Lovell (1936). We assume that all free paths start at the surface, and that the effective free path is the average of all free paths of a given electron, the free path being taken as the distance to the next intersection with the surface or the ordinary free path, whichever is least. Assuming further that the density of electrons travelling inwards from the surface is unifom (diffuse scattering), we have, if $\theta$ is the angle between the direction of motion and the normal to the film surface,

$$
l_{\mathrm{eff}}=\int_{0}^{\theta_{0}} \frac{a}{\cos \theta} \sin \theta \mathrm{d} \theta+\int_{\theta_{0}}^{\pi / 2} l \sin \theta \mathrm{d} \theta,
$$

where $\cos \theta_{0}=a / l$. This gives

$$
l_{\mathrm{eff}}=a\left(\log \frac{1}{a}+1\right),
$$

which is in qualitative agreement with the exact result when $l \gg a$. Examination of the integrals shows that the main contribution to $l_{\text {eff }}$ when $l \gg a$ comes from values of $\theta$ close to $\theta_{0}$; the dominant contribution to the current in films, however thin compared with the free path, therefore arises from electrons which, after reflection at the surface of the film, move in directions so nearly parallel to the surface that their free path remains of the order of the bulk free path. In the case of wires the constriction of free paths due to the geometry is more severe, and such electrons do not play any appreciable part.

\subsection{Comparison with experiment}

It was known already at the end of the nineteenth century that very thin films of metal exhibit a higher electrical resistivity than the same metal in bulk, and J. J. Thomson (1901) was the first to suggest that the effect arose from the limitation of the free path of the electrons and to give an approximate theory; he derived a formula similar to (37). Since then an enormous amount of experimental work has been carried out on the electrical properties of thin films; we mention in particular only Lovell's careful measurements on films of the alkali metals (Lovell 1936), and refer the reader for further information and references to the critical review by Chambers (1951). However, as we have already emphasized, if the measurements are to give information about the electronic parameters characteristic of the bulk metal, great care must be taken to eliminate all effects which may be peculiar to thin specimens other than the pure geometrical limitation of the free path with which the theory is alone concerned. In particular, both strain and impurity may be expected to play an increasing part in determining the phenomena as the size of the specimen is reduced. In fact it was not until recently that Andrew, working with relatively thick films at liquid-hydrogen and liquid-helium temperatures, obtained data which could be compared with any confidence with the theoretical predictions (Andrew 1949).

For thin wires there is much less experimental work than for films. The most important earlier experiments in this field are those of Eucken and Förster (1934) and succeeding workers, and data on wires were also obtained by Andrew in his recent work. To illustrate the kind of information that can be obtained from the experiments, Andrew's results on both films and wires are compared with the theory below.

There are two ways in which the ratio $a / l$ may be varied in carrying out the experiments. We may firstly use a single specimen and vary $l$ by changing the temperature. Since the bulk conductivity $\sigma_{0}$ cannot be measured on the specimen 
itself, it must then be assumed that the temperature variation of $\sigma_{0}$, and hence of $l$, is the same for a thin specimen as for a bulk specimen. Alternatively (and this was Andrew's procedure) $\sigma$ may be measured at one or more fixed temperatures for a number of specimens of different thickness or diameter; it is then important to ensure that the bulk free path is the same for all specimens.

2.4.1. Figure 1 shows Andrew's results on the resistance of rolled foils of tin at $3.8^{\circ} \mathrm{K}$. The resistance, expressed as a fraction of the value at $291^{\circ} \mathrm{K}$, is plotted logarithmically against the foil thickness, which ranges from 3 to $2000 \mu$.

In comparing these results with the theory, it is necessary to assume a value for the surface reflection coefficient $p$. Such evidence as exists mostly tends to show that the scattering is diffuse, so that $p=0$ (compare sections 2.4.1.1 and 4.6.4 below), and this was the value adopted by Andrew; the theoretical curve of $\sigma_{0} / \sigma$ for a film as a function of $a / l$ can then be fitted directly to the experimental results at constant temperature, and the full curve in figure 1 has been drawn in this way. The rather large scatter of the observations about the theoretical curve is probably due to a variation in residual resistivity between the specimens, but on the whole the agreement between theory and experiment is satisfactory. The process of fitting gives directly the value of the free path $l$ corresponding to the temperature of the experiment and the ratio of the bulk conductivity $\sigma_{0}$ to the (known) roomtemperature value. The results are that $l=9.5 \times 10^{-3} \mathrm{~cm}$ at $3.8^{\circ} \mathrm{K}$, and $\sigma_{0} / l=$ $4.5 \times 10^{22}$ gaussian units. Measurements at higher temperatures confirm that the value of $\sigma_{0} / l$ is constant, and with this value equation (4) gives $n=1.6 \times 10^{22}$. The number of atoms per unit volume, $n_{a}$, is $3.7 \times 10^{22}$ for tin, and thus $n / n_{a}=0.43$. Such a value for the number of electrons per atom is entirely reasonable, although in a complicated metal such as tin the precise figure has little significance.

2.4.1.1. Andrew's measurements on wires were carried out using mercury, and the results for $3.5^{\circ} \mathrm{K}$ and $2.5^{\circ} \mathrm{K}$ are shown in figure 2 . The exact analysis of conduction in thin wires had not been given at the time of Andrew's experiments, and the full curves in figure 2 were obtained on the basis of an approximate theory

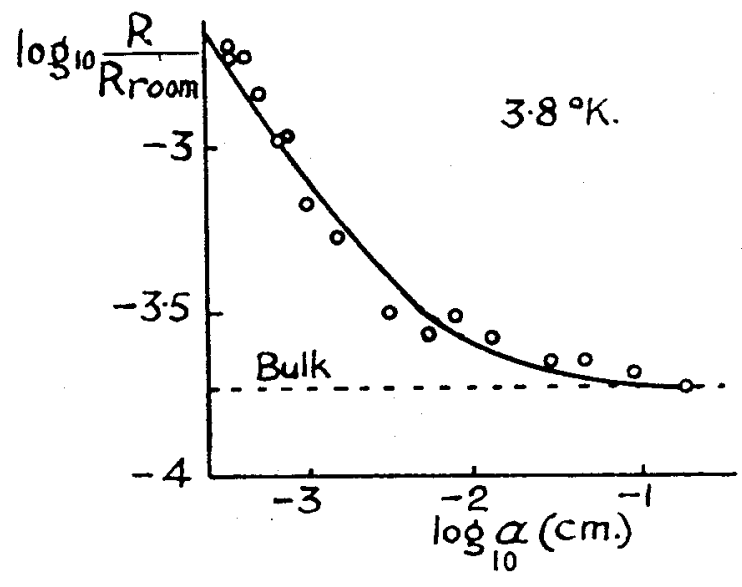

Figure 1. Experimental results on the resistivity of tin foils at $3.8^{\circ} \mathrm{K}$, plotted logarithmically against the foil thickness. The full line is the theoretical curve for diffuse scattering $(p=0)$. 


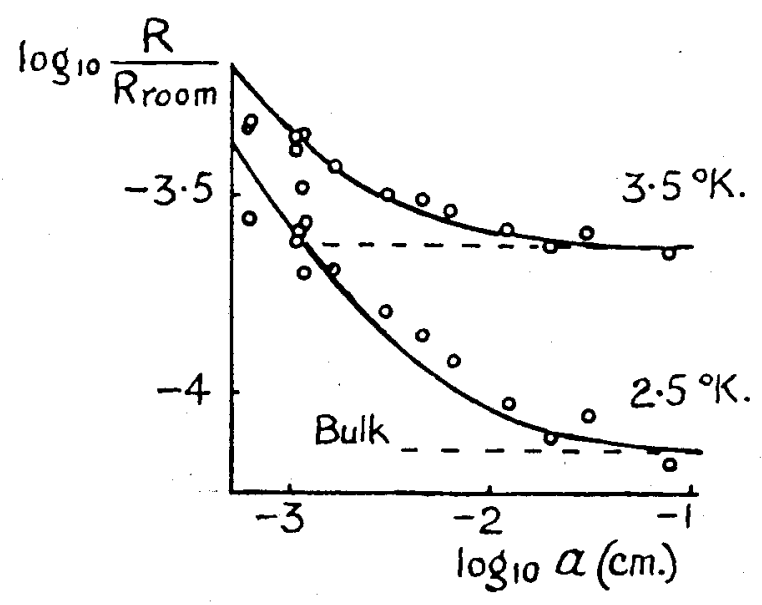

Figure 2. Experimental results on the resistivity of mercury wires at $3.5^{\circ} \mathrm{K}$ and $2.5^{\circ} \mathrm{K}$, plotted logarithmically against the wire diameter. The full lines are theoretical curves for diffuse scattering.

similar to Lovell's. Dingle (1950) later re-examined Andrew's results and found that the exact theory did not substantially alter the conclusions. $\dagger$ Dingle considered in particular the question of the value to be assigned to $p$; he found that, if a non-zero value is assumed, agreement with experiment could only be obtained by supposing that $p$ increases slightly with temperature. This is not plausible on theoretical grounds, since the various mechanisms which may lead to diffuse surface scattering, such as the thermal vibration of the surface atoms or the number of mobile surface defects of various kinds, would be expected to become more effective the higher the temperature, causing $p$ to decrease. The value $p=0$ is therefore the most likely; it must be emphasized, however, that more experimental and theoretical work is required before the question can be regarded as definitely settled (compare also section 4.6.4.1). Adopting the value $p=0$ and disregarding the results obtained for the thinnest wires used in the experiments, which do not fit in with the theoretical predictions, it is found that $l=5.6 \times 10^{-3} \mathrm{~cm}$ at $2.5^{\circ} \mathrm{K}$ and $a_{0} / l=2.5 \times 10^{22}$, which corresponds to 0.15 electrons per atom in mercury.

2.4.2. Corresponding experiments have been carried out by MacDonald and Sarginson (1950) on thin wires of very pure sodium; such experiments are of particular interest, since for an alkali metal quantitative agreement with the freeelectron theory is to be expected. MacDonald and Sarginson compare their results with the theory developed by them for a square wire, using values of $l$ estimated on the basis of the free-electron theory from the observed conductivity of a bulk sample. $\left(l \approx 4.5 \times 10^{-3} \mathrm{~cm}\right.$ at $4.2^{\circ} \mathrm{K}$.) The agreement between theory and experiment is only fair, and MacDonald and Sarginson conclude that the hypothesis of entirely diffuse scattering at the boundaries is not generally fulfilled for their specimens, and that the

$\dagger$ It is worth noting that the simple formula $\sigma_{0} / \sigma=1+1 / \kappa$ gives values of $\sigma_{0} / \sigma$ which differ from the exact values (for $p=0$ ) by less than $5 \%$ over the whole range of $\kappa$. This formula (or a slight generalization of it) is associated with the name of Nordheim, and was used in the evaluation of the earlier experimental work on thin wires (Nordheim 1934, Eucken and Förster 1934). 
degree of elastic scattering is a function of the specimen size. Further experiments on sodium are considered in sections 3.2 and 3.3 .

\subsection{The 'kinetic theory' solution of thin-conductor problems}

Chambers (1950a) has recently given an elegant formulation of the theory of thin-conductor problems, in which he writes down the solution of the Boltzmann equation in a form suggested by simple kinetic theory considerations.

In the presence of an electric field $E$ in the $x$-direction, and with $f=f_{0}+f_{1}(\mathbf{v}, \mathbf{r})$, the Boltzmann equation takes the form

$$
\mathbf{v} \cdot \operatorname{grad}_{\mathbf{r}} f_{1}-\frac{E \epsilon}{m} \frac{\partial f_{0}}{\partial v_{x}}=-\frac{f_{1}}{\tau} .
$$

A particular solution is

$$
f_{1}(\mathbf{v}, \mathbf{r})=\frac{\epsilon \tau E}{m} \frac{\partial f_{0}}{\partial v_{x}}\left\{1-\exp \left(-\frac{\left|\mathbf{r}-\mathbf{r}_{0}\right|}{\tau v}\right)\right\},
$$

where $\mathbf{r}-\mathbf{r}_{0}$ is a vector parallel to $\mathbf{v}$. This solution applies to conductors of arbitrary shape and, if $\mathbf{r}=\mathbf{r}_{0}$ is taken to be a point on the surface of the conductor, it satisfies the boundary conditions corresponding to diffuse scattering at the surface.

Equation (39) may be derived from first principles as follows. Consider electrons passing through a point $\mathbf{r}$ in the metal in the direction $\mathbf{r}_{0}-\mathbf{r}$. The probability that an electron will travel a distance $s$ and then suffer a collision between $s$ and $s+d s$ is $e^{-s / l} \mathrm{~d} s / l$, but electrons which arrive at $\mathbf{r}_{0}$ will certainly collide there if the scattering is diffuse. The mean distance travelled by an electron without collision after passing through $\mathbf{r}$ is therefore

$$
l^{\prime}=\int_{0}^{\left|\mathbf{r}-\mathbf{r}_{0}\right|} s e^{-s / l} \mathrm{~d} s / l+\int_{\left|\mathbf{r}-\mathbf{r}_{0}\right|}^{\infty}\left|\mathbf{r}-\mathbf{r}_{0}\right| e^{-s / l} \mathrm{~d} s / l=l\left(1-e^{-\left|\mathbf{r}-\mathbf{r}_{0}\right| / l}\right) ;
$$

also, for electrons travelling in the opposite direction $\mathbf{r}-\mathbf{r}_{0}$, the mean distance travelled without collision before reaching $\mathbf{r}$ is given by the same expression. The mean drift velocity acquired by these electrons in the electric field is therefore

$$
\Delta v_{x}=\frac{\epsilon E l^{\prime}}{m v}=\frac{\epsilon \tau E}{m}\left(1-e^{-\left|\mathbf{r}-\mathbf{r}_{0}\right| / l}\right),
$$

and $f_{1}$ which is the change due to the presence of the field in the number of electrons travelling in the direction $\mathbf{r}-\mathbf{r}_{0}$ with velocity $\mathbf{v}$, is given by

$$
f_{1}(\mathbf{v}, \mathbf{r})=\frac{\partial f_{0}}{\partial v_{x}} \Delta v_{x}=\frac{\epsilon \tau E}{m} \frac{\partial f_{0}}{\partial v_{x}}\left(1-e^{-\left|\mathbf{r}-\mathbf{r}_{0}\right| / l}\right),
$$

in agreement with equation (39).

2.5.1. The current density may now be calculated exactly as in section 2.1.1, except that the appropriate polar coordinate system $(v, \delta, \varpi)$ to be used here has the $x$-axis as polar axis, this being the only preferred direction which occurs in the present general formulation of the theory. The effective conductivity $\sigma$ is obtained by integrating the current density over the cross-sectional area $S$ of the conductor, and the ratio of $\sigma$ to the bulk conductivity $\sigma_{0}$ may be written in the compact form

$$
\frac{\sigma}{\sigma_{0}}=1-\frac{3}{4 \pi S} \int_{S} \mathrm{~d} S \int_{0}^{2 \pi} \mathrm{d} \varpi \int_{0}^{\pi} \mathrm{d} \delta \sin \delta \cos ^{2} \delta e^{-\left|\mathbf{r}-\mathbf{r}_{0}\right| / l}
$$


To evaluate $\sigma / \sigma_{0}$ for any shape of conductor it is necessary to express $\left|\mathbf{r}-\mathbf{r}_{0}\right|$ in terms of the position of the point $\mathbf{r}$ and the angles $\delta$ and $\varpi$ which define the direction of $\mathbf{r}-\mathbf{r}_{0}$, and to carry out the integrations. For example, the expressions (15) and (28) given above for a thin film and a thin wire may readily be derived in this way; for a film, however, it is convenient first to change back to the polar coordinates $(v, \theta, \phi)$ used in section 2.1.1. A more complicated problem, which well illustrates the power of the present method, is provided by the case of a thin wire placed in a longitudinal magnetic field; this is discussed in section 3.2.

The case of partially elastic scattering has also been considered by Chambers, who derived the appropriate generalization of (43); in this case, however, it is impossible to obtain a simple formula applicable to conductors of arbitrary crosssection.

\section{Magnetic effects in thin conductors}

3.1. In considering conduction in thin specimens when a magnetic field is present, we shall confine attention to the film and the circular wire, and to purely longitudinal or transverse fields. There are then altogether five possible arrangements: these fall into three groups, depending on whether the magnetic field is parallel or perpendicular to the electric current, and whether the Hall electric field which is set up in the latter case is or is not uniform across the specimen.

(A) $H$ parallel to $J$. No Hall field is produced, and this considerably simplifies the analysis. There are two cases in this group:

(A1) the wire with $H$ and $J$ parallel to the axis (section 3.2), and

(A2) the film with $H$ and $J$ parallel to each other and in the plane of the film.

(B) $H$ perpendicular to $J$ : Hall field uniform. The Hall field is set up in the direction perpendicular to both $H$ and $J$ to prevent any current flow in this direction. If it is developed across a 'thin' dimension of the specimen it will vary in magnitude across the specimen and its determination as a function of position presents considerable difficulties (see section 3.5). In one case, however, namely that of a thin film with $H$ perpendicular to the plane of the film, the Hall field (being in the plane of the film) is developed across a 'long' dimension of the specimen and is consequently constant. This case is in fact very simple to analyse (section 3.4).

(C) $H$ perpendicular to $J$ : Hall field non-uniform. The two remaining cases fall into this group: they are

(C1) the film with $H$ in the plane of the film but perpendicular to $J$, and (C2) the wire with $H$ perpendicular to the axis.

An approximate treatment has been given of case (C1) (section 3.5); case (C2) has not yet been considered, and indeed is of such complexity as to be virtually insoluble.

Exact treatments have so far been given only of cases (A1) and (B), and these will be considered in some detail below. Experimental data are so far available only for wires (cases (A1) and (C2)); the case (A1) of a thin wire in a longitudinal magnetic field is thus of particular interest as being the only case at present for which there exist both an exact evaluation of the theory and experimental results with which to compare it. 
A thin-conductor effect of the present type was first observed by MacDonald (1949). The ordinary bulk magneto-resistance effect always leads to an increase in resistance; MacDonald found, however, that the resistance of a thin sodium wire decreased with $H$ in a longitudinal field, whilst in a transverse field it increased initially, passed through a maximum and then decreased. Sodium has also been used in subsequent investigations (MacDonald and Sarginson 1950, Chambers 1950a); this metal (and possibly pure lithium and potassium) appears unfortunately to be the only metal suitable for the experiments in view of the higher inherent bulk magnetoresistance of all other metals, including even the heavier alkali metals (MacDonald 1950). The bulk effect is greatest under just the same conditions of low temperatures and high fields as are required to observe the free electron phenomena, and in general it obliterates the latter entirely.

\subsection{The conductivity of thin wires in a longitudinal magnetic field}

MacDonald, at the time of his discovery of the effect, correctly interpreted its physical origin, and in particular he explained the simple decrease in resistance which occurs in a wire in a longitudinal field as being due to the lessened infuence of scattering at the walls of the wire when the electrons are forced to pursue spiral paths around the lines of force of the magnetic field.

An exact analysis of this case (A1) has been given by Chambers (1950a), assuming diffuse scattering at the boundaries. When the electric and magnetic fields are parallel, the magnetic force on the electrons is always perpendicular to the electric force; we can then regard the electric field alone as producing a drift current in the usual way, and the magnetic field simply as modifying the electronic trajectories. The solution of the Boltzmann equation is therefore formally the same as in the absence of a magnetic field (equation (42)), and the conductivity is still given by (43), provided that $\left|\mathbf{r}-\mathbf{r}_{0}\right|$ is replaced by the distance $s$ from the point $\mathbf{r}$ to the point on the surface $\mathbf{r}_{0}$ measured along the curved trajectory of the electrons.

3.2.1. The integral (43) was evaluated by Chambers for this case by means of an ingenious series of transformations. With a magnetic field $H$ along the $x$-axis (the axis of the wire), electrons travelling at an angle $\delta$ to the $x$-axis will move in helical paths whose projections on the $y z$-plane are circles of radius

$$
r=m \bar{v} c \sin \delta / \epsilon H=r_{0} \sin \delta .
$$

If now, while an electron is travelling from $\mathbf{r}_{0}$ to $\mathbf{r}$, the projection of its path on the $y z$-plane traverses an angle $\psi$ around such a circle, then the projection of the distance $s$ on the $y z$-plane is $\psi r_{0} \sin \delta$, so that $s=\psi r_{0}$. Equation (43) therefore becomes

$$
\frac{\sigma}{\sigma_{0}}=1-\frac{3}{4 \pi S} \int_{S} \mathrm{~d} S \int_{0}^{2 \pi} \mathrm{d} \varpi \int_{0}^{\pi} \mathrm{d} \delta \sin \delta \cos ^{2} \delta e^{-r_{0} \psi / l},
$$

where $\psi=\psi(y, z, \delta, \varpi)$.

3.2.2. If we consider a fixed value of the azimuth angle $\varpi$ and perform the integrations over $S$ and $\delta$, it is evident by symmetry that the result must be

$\dagger r$ and $r_{0}$ in this equation must not, of course, be confused with the magnitudes of the position vectors $\mathbf{r}$ and $\mathbf{r}_{0}$ 


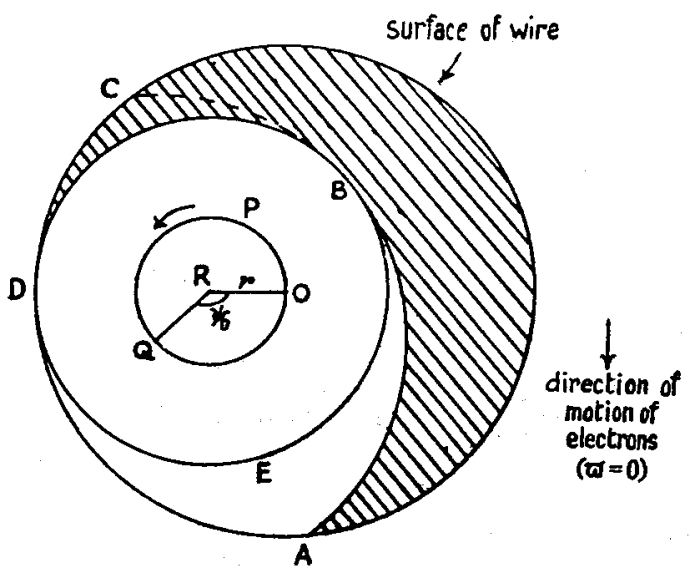

Figure 3. Construction for the determination of $P\left(\gamma, \psi_{0}\right)$ (see text).

independent of $\varpi$; we may therefore restrict ourselves to a particular value of $\varpi$, say $\varpi=0$. We may furthermore confine ourselves to values of $\delta$ in the range $\left(0, \frac{1}{2} \pi\right)$ and write (45) in the form

$$
\frac{\sigma}{\sigma_{0}}=1-\frac{3}{S} \int_{0}^{\pi / 2} \mathrm{~d} \delta \sin \delta \cos ^{2} \delta \int_{S} \mathrm{~d} S\left(e^{-r_{0} \psi / l}\right)_{\varpi=0} .
$$

The integrand of (46) refers to electrons which, since colliding with the wall, have turned through angles between $\psi$ and $\psi+\mathrm{d} \psi$ and are, at the instant considered, travelling in the direction $(\delta, \varpi=0)$. If we denote the proportion of the total crosssection of the wire occupied by these electrons by $p(\gamma, \psi) \mathrm{d} \psi$ (where $\gamma$ is defined below), we may rewrite (46) as

$$
\frac{\sigma}{\sigma_{0}}=1-3 \int_{0}^{\pi / 2} \mathrm{~d} \delta \sin \delta \cos ^{2} \delta \int_{0}^{2 \pi} p(\gamma, \psi) e^{-r_{0} \psi / l} \mathrm{~d} \psi .
$$

It remains to find an expression for $p(\gamma, \psi)$, which is a function only of $\psi$ and of the ratio of the radius $\frac{1}{2} a$ of the wire $\uparrow$ to the radius $r=r_{0} \sin \delta$ of the projection of the orbit, i.e. of $\gamma=2 r / a=2 \sin \delta / \beta$, where $\beta=a / r_{0}$.

Chambers has given the following construction for the determination of $p(\gamma, \psi)$ (figure 3). Suppose, for example, that $r \leqslant \frac{1}{2} a(\gamma \leqslant 1)$, and consider electrons which traverse the projection of their orbit in the anti-clockwise direction and which are travelling vertically downwards at the instant considered. Let $O$ be the centre of the wire, and let $R$ be chosen such that $O R$ is horizontal and of length $r$. With centre $R$, draw the circles $O P Q$ of radius $r$ and $B D E$ of radius $\frac{1}{2} a-r$. The circle $B D E$ is then such that, for the group of electrons considered, no point within it can be reached by a trajectory starting from the wall. Supose that $O Q$ subtends an angle $\psi_{0}$ at $R$, and with centre $Q$ and radius $\frac{1}{2} a$ draw the circular arc $A B C$ which touches the circle $B D E$ at $B$; it is easily seen that this is the locus of the positions of all electrons of the kind considered which have turned through an angle $\psi_{0}$ since colliding with the wall. The portion $B C$ must be excluded, however, since it corresponds to electrons whose 
trajectory lies partly outside the wire; if, instead, we continue with the arc $B D$ of the circle $B D E$, then the shaded area between $A B D$ and the surface of the wire contains all those electrons which have turned through angles $\psi \leqslant \psi_{0}$. The shaded area is therefore $P\left(\gamma, \psi_{0}\right)=\int_{0}^{\psi_{0}} p(\gamma, \psi) \mathrm{d} \psi \cdot p(\gamma, \psi)$ may be obtained from this by differentiation or, alternatively, (47) may be expressed directly in terms of $P(\gamma, \psi)$ by partial integration.

Analytical expressions for $p(\gamma, \psi)$ may be obtained by considering the geometry of figure 3 and the corresponding figure for $\gamma>1$. It is readily shown that, for $\gamma \leqslant 1$,

$$
\pi p(\gamma, \psi)=\gamma(1-\gamma)=\gamma^{2} \cos ^{2} \frac{1}{2} \psi+\gamma \cos \frac{1}{2} \psi\left(1-\gamma^{2} \sin ^{2} \frac{1}{2} \psi\right)^{1 / 2} \quad(0 \leqslant \psi \leqslant 2 \pi),
$$

and, for $\gamma \geqslant 1$,

$$
\left.\begin{array}{rlrl}
\pi p(\gamma, \psi) & =2 \gamma \cos \frac{1}{2} \psi\left(1-\gamma^{2} \sin ^{2} \frac{1}{2} \psi\right)^{1 / 2} & & \left(0 \leqslant \psi \leqslant 2 \sin ^{-1}(1 / \gamma)\right), \\
& =0 & & \left(2 \sin ^{-1}(1 / \gamma)<\psi \leqslant 2 \pi\right),
\end{array}\right\}
$$

where the positive value of the square root is to be taken.

Note that, for $\beta \geqslant 2, \gamma \leqslant 1$ for all $\delta$, while for $\beta<2$, we have $\gamma<1$ for small $\delta$ and $\gamma>1$ for large $\delta\left(\sim \frac{1}{2} \pi\right)$. Physically this means simply that for strong enough fields $(\beta \geqslant 2)$ all electronic trajectories are curved into paths of radius less than the wire radius; for smaller fields, those electrons travelling at small angles to the axis and therefore having small transverse velocities will still follow such paths, but electrons moving at greater angles to the axis will follow paths of radius greater than the wire radius.

3.2.3. Values of $\sigma / \sigma_{0}$ may now be computed by graphical integration using figure 3, or by approximate analytical methods using equations (48) and (49). The final results, as is clear from (47), depend only on the two dimensionless parameters $\beta=a / r_{0}$ and $\kappa=a / l$, which are measures of the strength of the magnetic field and of

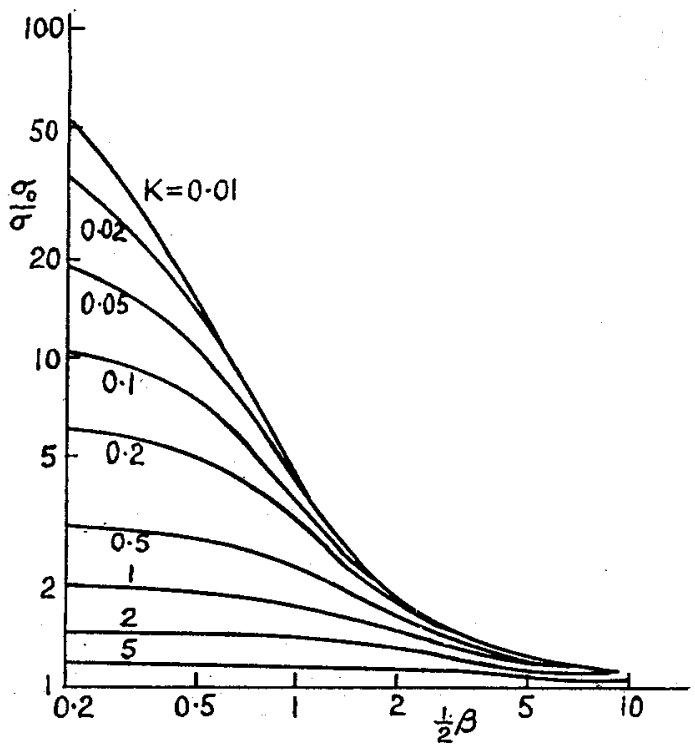

Figure 4. Theoretical conductivity of thin wires in a longitudinal magnetic field. 
the free path respectively. In figure 4 theoretical values of the resistivity ratio $\sigma_{0} / \sigma$ are shown plotted against $\frac{1}{2} \beta$, for various values of $\kappa$. The values for $\beta=0$ are those given by Dingle (section 2.2), and, as is to be expected on physical grounds, the resistance decreases steadily as $\beta$ increases and tends to the bulk value as $\beta$ tends to infinity.

\subsubsection{Comparison with experiment}

If we plot $\kappa \sigma_{0} / \sigma$ against $\frac{1}{2} \beta$ for various $\kappa$, then, since $\kappa=a / l$ and $\sigma_{0} / l$ is independent of the temperature, the ordinates are proportional to the resistivity $1 / \sigma$, and, since $\frac{1}{2} \beta=\epsilon a H /(2 m \bar{v} c)$, the abscissae are proportional to the applied magnetic field. The theoretical curves of $\kappa \sigma_{0} / \sigma$ against $\frac{1}{2} \beta$, plotted logarithmically, are therefore directly superposable on the experimental curves of $1 / \sigma$ against $H$ at a number of temperatures, and the proportionality constants give values of $\sigma_{0} / l$ and $m \bar{v}$ directly. The advantage of using a magnetic field is that all the required information can be obtained from one specimen only, and it is not necessary to assume, as in zero-field experiments, that the free path in a thin specimen is the same as in a bulk specimen.

Figure 5 shows experimental results obtained by Chambers on a sodium wire of diameter $30 \mu$ at various temperatures; also shown are the theoretical curves for $\kappa=0.5,1$ and 2 , adjusted to fit as well as possible. It has, of course, been assumed that the hypothesis of diffuse reflection at the surface is correct, and that the bulk magneto-resistance effect is negligible. The departure from theory in high fields is, in fact, due to the onset of the bulk effect, but this does not interfere seriously with the comparison between theory and experiment. The results of the comparison are that, in sodium, $\sigma_{0} / l=8.1 \times 10^{22}$ and $m \bar{v}=9.1 \times 10^{-20}$; these values provide two independent estimates of the number of conduction electrons, and agree reasonably well with the values

$$
\sigma_{0} / l=6.3 \times 10^{22} \text { and } m \bar{v}=9.7 \times 10^{-20}
$$

obtained from (4) and (1a) assuming one conduction electron per atom.

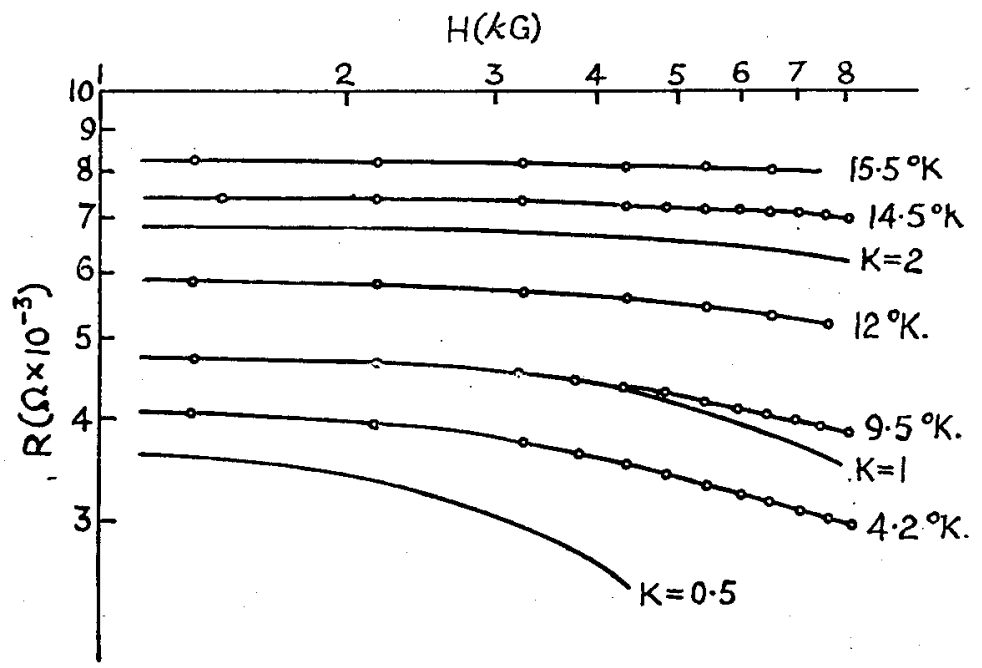

Figure 5. Experimental results on a $30 \mu$ sodium wire in a longitudinal magnetic field at various temperatures, with theoretical curves. 


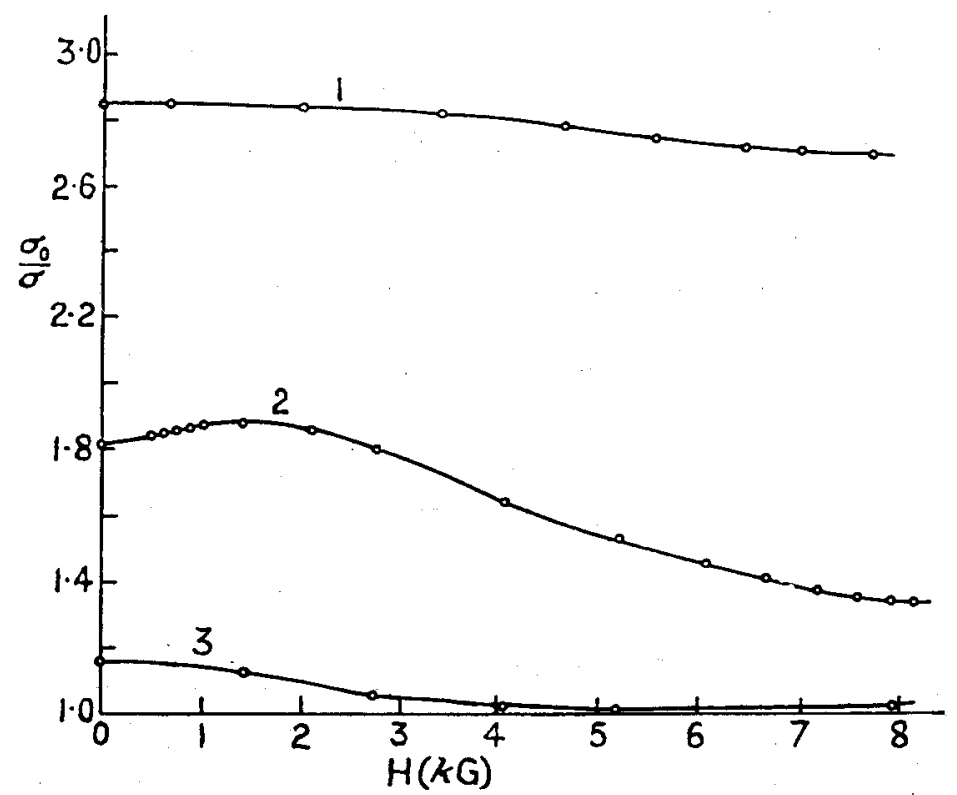

Figure 6. Experimental results on sodium wires of various diameters in a transverse magnetic field. Diameters: $1,20 \mu ; 2,30 \mu ; 3,66 \mu$. Temperature $=4.2^{\circ} \mathrm{K}$.

3.3 Similar experiments have been reported by MacDonald and Sarginson (1950) on sodium wires in both longitudinal and transverse magnetic fields, and some typical results for transverse fields are shown in figure 6; note particularly that in this case the resistance may increase initially with $H$. Detailed theoretical analysis of this arrangement (C2) is prohibitively difficult, and MacDonald and Sarginson compared their results with an approximate theory developed by them for the somewhat simpler thin-film arrangement (C1) (see section 3.5), with which they are in qualitative agreement.

\subsection{The conductivity of thin films in a transverse magnetic field}

The case (B) of a thin film in a magnetic field which is perpendicular to the plane of the film has been analysed by Sondheimer (1950). In this case it is not immediately obvious from simple physical arguments how the presence of the magnetic field affects the conductivity at all. The formal analysis, however, presents no difficulties and is a straightforward generalization of the treatment given in section 2.1 for a film in zero magnetic field; it leads to the interesting result that the conductivity is an oscillatory function of the strength of the applied magnetic field.

3.4.1. We use the notation of section 2.1, but suppose now that the film is subjected to an electric field $\left(E_{x}, E_{y}, 0\right)$ in the plane of the film and a magnetic field $(0,0, H)$. For this particular arrangement, the condition curl $\mathbf{E}=0$ ensures that the electric field components are constant across the thickness of the film. The Boltzmann equation takes the form

$$
\frac{\partial f_{1}}{\partial z}+\frac{f_{1}}{\tau v_{z}}-\frac{\epsilon H}{m c v_{z}}\left(v_{y} \frac{\partial f_{1}}{\partial v_{x}}-v_{x} \frac{\partial f_{1}}{\partial v_{y}}\right)=\frac{\epsilon}{m v_{z}}\left(E_{x} \frac{\partial f_{0}}{\partial v_{x}}+E_{y} \frac{\partial f_{0}}{\partial v_{y}}\right),
$$


which is the generalization of equation (9). To solve this equation we put

$$
f_{1}=\left(v_{x} c_{1}+v_{y} c_{2}\right) \frac{\partial f_{0}}{\partial v}
$$

where $c_{1}$ and $c_{2}$ do not depend explicitly on $v_{x}$ and $v_{y}$. With this form for $f_{1}$, equation (50) leads to two simultaneous equations for $c_{1}$ and $c_{2}$; if we introduce the complex quantities $g=c_{1}-i c_{2}, \mathcal{F}=E_{x}-i E_{y}$, we may write these equations in the compact form

$$
\frac{\partial g}{\partial z}+g\left(\frac{1}{\tau v_{z}}+\frac{i \epsilon H}{m c v_{z}}\right)=\frac{\epsilon \mathcal{F}}{m v v_{z}},
$$

and the problem is now formally identical with that of section 2.1.

3.4.2. The rest of the calculation is entirely analogous to that of section 2.1 , and the result may be expressed as follows. We define a complex conductivity $\sigma_{c}$ by means of the relation $\overline{\mathcal{J}}=\sigma_{c} \mathcal{F}$, where $\overline{\mathcal{J}}=\bar{J}_{x}-i \bar{J}_{y}$ is the complex current density averaged in the usual way across the thickness of the film. We further introduce the parameters $\kappa=a / l, \beta=a / r_{0}$ ( $a$ being the thickness of the film), and the complex variable $s=\kappa+i \beta$. We then have, as the generalization of (16),

$$
\frac{\sigma_{0}}{\sigma_{c}}=\frac{\Phi(s)}{\kappa},
$$

where the function $\Phi$ is given as before by (17) when $p=0$ and by (25) when $p \neq 0$, but is now a function of a complex variable. (We may remark here that the present problem is the only one of the magnetic-field problems which can easily be solved for the case $p \neq 0$.)

3.4.3. Equation (53) contains all the results required for comparison with experiment. The ordinary electrical conductiviy $\sigma$, for example, is measured by applying an electric field in, say, the $x$-direction and observing the current in this direction, no electric current being allowed to flow in the transverse direction. We therefore have $\sigma=\mathcal{R}(\overline{\mathcal{J}}) / \mathcal{R}(\mathcal{F})$, with $\mathcal{I}(\overline{\mathcal{J}})=0$, where $\mathcal{R}$ and $\mathcal{I}$ denote the real and imaginary parts respectively; eliminating the transverse electric field, we readily find that

$$
\frac{\sigma_{0}}{\sigma}=\sigma_{0} \mathcal{R}\left(\frac{1}{\sigma_{c}}\right)=\mathcal{R}\{\Phi(s)\} / \kappa .
$$

The Hall coefficient is defined by $A_{\mathrm{H}}=E_{y} / H \bar{J}_{x}$, where $E_{y}$ is the transverse electric field set up under the above experimental conditions, and we easily obtain

$$
A_{\mathrm{H}} / A_{\mathrm{H}, 0}=\mathcal{I}\{\Phi(s)\} / \beta,
$$

where $A_{\mathrm{H}, 0}=-1(n \epsilon c)$ is the Hall coefficient of the bulk metal.

3.4.4. In the limit of zero magnetic field $(\beta=0)$, equation (54) for the conductivity reduces to equation (16), and (55) gives a corresponding expression for the Hall coefficient of a thin film in a vanishingly small magnetic field. In this limit $A_{\mathrm{H}} / A_{\mathrm{H}, 0}$ depends only on $\kappa$, and is shown graphically in figure 7 for three values of $p$; it is seen that the Hall coefficient of a thin film shows an increase above 


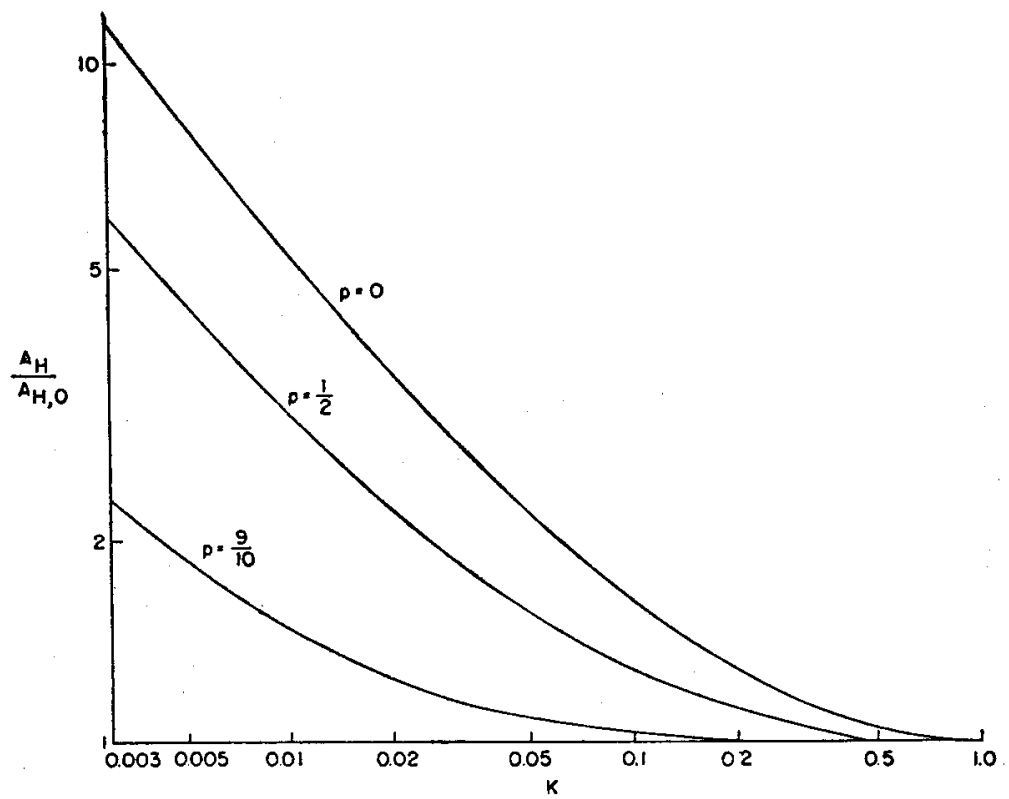

Figure 7. Theoretical Hall coefficient of thin metallic films (in small magnetic fields) divided by the Hall coefficient of the bulk metal.

the bulk value analogous to the increase of the electrical resistivity. For very small $\kappa$, we find that

$$
\frac{A_{\mathrm{H}}}{A_{\mathrm{H}, 0}}=\frac{4}{3} \frac{1-p}{1+p} \frac{1}{\kappa\{\log (1 / \kappa)\}^{2}},
$$

which shows that the Hall coefficient increases less rapidly than the resistance (compare (27)).

3.4.5. The field variation of the resistivity may be computed from (54), and, as mentioned above, it is found that the resistance oscillates with the strength of the applied magnetic field. Figure 8 shows some typical curves for $p=0$ and various values of $\kappa$. In general the resistance increases initially with $H$ and reaches its first maximum when $\beta$ is approximately unity; the higher oscillations are of roughly constant spacing but decrease rapidly in amplitude, and in very strong fields the resistance tends to the constant asymptotic value

$$
\left(\frac{\sigma_{0}}{\sigma}\right)_{\beta=\infty}=1+\frac{3}{8 \kappa}(1-p)
$$

which, for small $\kappa$, is greater than the value in zero field. The oscillations die out and the resistance tends towards the bulk value as $\kappa$ becomes large or as $p$ tends to unity; the position of the maxima and minima, however, is almost independent of $\kappa$ and $p$, and $\beta$ may thus be estimated unambiguously by observing the value of $H$ for which, for example, the first maximum is reached (assuming, however, as usual that the bulk magneto-resistance effect is negligible).

The Hall coefficient, on the other hand, does not oscillate with $H$, and decreases steadily towards the bulk value as $H$ increases to infinity. 


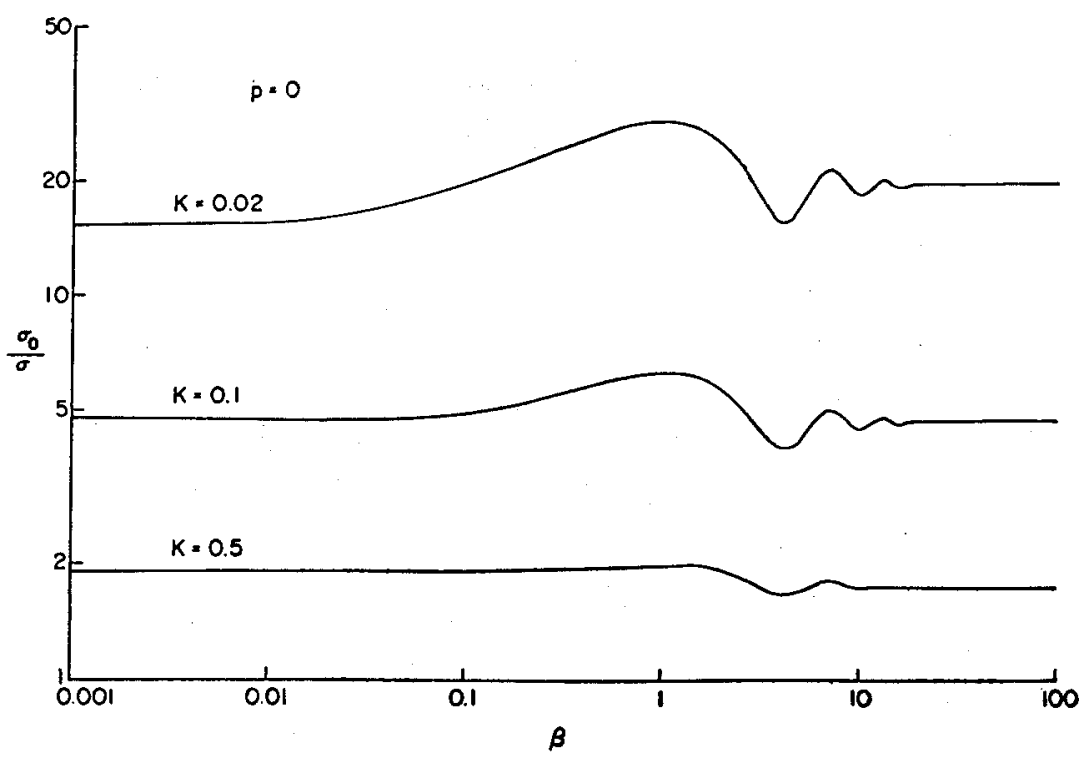

Figure 8. Theoretical variation of resistance of thin metallic films with magnetic field perpendicular to surface of film, assuming diffuse surface scattering.

3.4.6. The resistance oscillations have been explained by Chambers (1950a) as being essentially due to the oscillations in speed of an electron moving in perpendicular electric and magnetic fields. The speed at time $t$ is a trigonometric function of $\epsilon H t / m c=\bar{v} t / r_{0}$, and this causes the distribution function $f_{1}(\mathbf{v}, z)$ to be a fluctuating function of $z / r_{0}$. The effect occurs only in thin films because the presence of the metal surface is required to provide a finite limit to the distance from which electrons can come to contribute to the current at $z$; when the thickness is large compared with the free path, the elementary oscillating contributions to $f_{1}(\mathbf{v}, z)$ add up to give a non-fluctuating total, and in fact $f_{1}$ is then independent of $z$.

Equation (57) shows that, in a strong magnetic field, the effective free path of the electrons (see section 2.3.2) is of the order of the thickness of the film. We therefore have the physically plausible result that a strong transverse magnetic field eliminates the contribution to the current of those electrons moving nearly parallel to the surface which are responsible in the absence of a field for the logarithmic dependence of $l_{\text {eff }}$ on the ratio $l / a$.

3.5. MacDonald and Sarginson (1950) have given an elaborate discussion of a thin film for the case (C1) where the magnetic field is in the plane of the film but perpendicular to the current. For the details of the analysis the reader is referred to the original paper. The Hall field is now in the $z$-direction (perpendicular to the plane of the film) and varies across the thickness of the film. It can, in principle, be evaluated as a function of $z$ and $H$ by solving a complicated integral equation which expresses the condition that the current density in the $z$-direction must vanish everywhere. MacDonald and Sarginson have evaluated the Hall field approximately for the case of small magnetic fields, and have found that it increases rapidly near the edges of the film but is approximately constant over most of the cross-section, as shown in figure 9. In their calculation of the conductivity MacDonald and Sarginson 


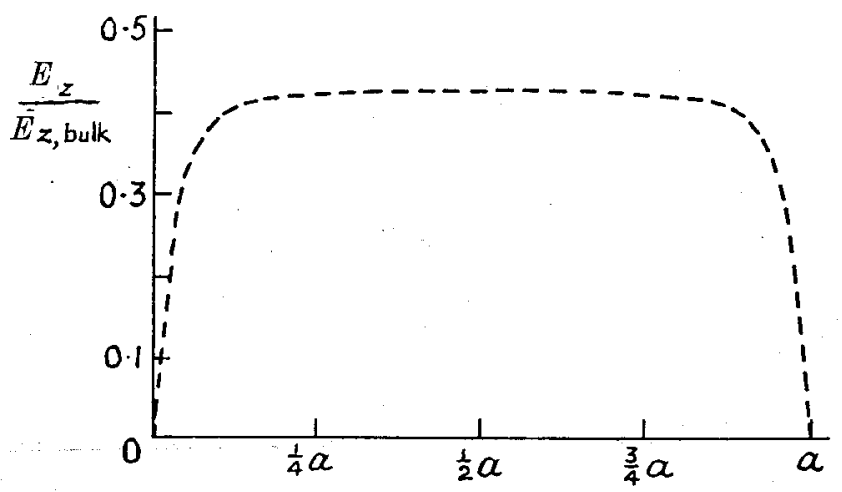

Figure 9. Theoretical variation of Hall field $E_{z}$ across a thin film of thickness $a=0.64 \times$ free path, assuming diffuse scattering at the boundaries

were forced, however, to assume for simplicity that the Hall field was constant over the whole cross-section. In view of this assumption the results must be treated with caution; they indicate that there are no oscillations, but that, for sufficiently thin films, the resistance increases initially with $H$, passes through a single maximum and then decreases steadily towards the bulk metal value.

3.6. The only magnetic-field experiments on thin films so far reported (MacDonald and Sarginson 1950) have been carried out on foils of silver, gold and tin. Although small indications of the effects discussed above were observed, it was found that the bulk magneto-resistance effect was the dominating factor in all cases, and no definite conclusions could be drawn. Experiments on thin films of sodium in magnetic fields both in and perpendicular to the plane of the film are highly desirable in order to test the theoretical predictions; at the same time the theory should be rounded off by a discussion of the third case (A2) where the magnetic field is in the plane of the film and parallel to the current.

\section{The anomalous skin effect}

4.1. H. London (1940) discovered that the resistivity of metals at high frequencies and very low temperatures was much greater than is predicted by the usual theory of the skin effect. He suggested that a free-path phenomenon was involved, but did not elaborate his idea. The problem was later taken up by Pippard (1947), who studied it in detail both experimentally and theoretically; using mainly physical arguments he succeeded in elucidating all the essential features involved. The quantitative theory of the effect is based on the methods discussed in the present article and is due to Reuter and Sondheimer (1948).

\subsection{The normal skin effect}

The basic concepts are best introduced by considering the theory of the skin effect in metals in its simplest form. Let the surface of a semi-infinite metal be the $x y$-plane and let the positive $z$-axis be directed towards the interior of the metal. The electric field $E(z) e^{i \omega t}$ is taken to be in the $x$-direction, $\omega$ being the angular frequency, and the magnetic field $H(z) e^{i \omega t}$ is in the $y$-direction; the factor $e^{i \omega t}$ which occurs in all timedependent quantities need not be written down explicitly and will in future be omitted. 
Neglecting the displacement current, Maxwell's equations take the form

$$
-H^{\prime}(z)=4 \pi J(z) / c, \quad E^{\prime}(z)=-i \omega H(z) / c,
$$

where $J(z)$ is the current density. Eliminating $H$, we obtain

$$
E^{\prime \prime}(z)=4 \pi i \omega J(z) / c^{2},
$$

and, combining this with Ohm's law in the form $J=\sigma_{0} E$, we have

$$
E^{\prime \prime}(z)=4 \pi i \omega \sigma_{0} E(z) / c^{2},
$$

so that

$$
\begin{gathered}
E(z)=E(0) e^{-k z}, \quad \text { with } \quad k=(1+i) \sqrt{2 \pi \omega \sigma_{0}} / c=(1+i) / \delta, \\
\delta=c / \sqrt{2 \pi \omega \sigma_{0}}
\end{gathered}
$$

being the depth of penetration of the field (the 'classical skin depth').

In high-frequency experiments the quantity most directly accessible to measurement is not the resistivity of the metal in the ordinary sense, but the surface resistivity $R$, which is the real part of the surface impedance $Z$. $Z$ is defined as the ratio of the electric field at the surface of the metal to the total current per unit area of surface, and thus

$$
Z=R+i X=E(0) / \int_{0}^{\infty} J(z) \mathrm{d} z,
$$

$X$ being the surface reactance. Equations (59) and (61) give

$$
R=X=\sqrt{2 \pi \omega / c^{2} \sigma_{0}},
$$

and $R$ and $X$ therefore vary directly as $\sqrt{ } \omega$ for a specimen at a given temperature, and inversely as $\sqrt{ } \sigma_{0}$ for a given frequency.

4.2.1. These formulae are in good agreement with observation on metals at room temperature for all wavelengths in the radio-frequency region. Pippard found, however, that as $\sigma_{0}$ is increased by lowering the temperature, the surface conductivity $1 / R$ increases less rapidly than is predicted by equation (64) and that, as $\sigma_{0}$ tends to infinity, $1 / R$ tends to a constant value which is different for each metal; the typical form of the curves is shown in figure 11 below. This is the phenomenon known as the anomalous skin effect, and it evidently involves a breakdown of the simple theory outlined above.

4.2.2. In the above form the theory is essentially phenomenological in character and makes no direct reference to electron theory; it is based only on Maxwell's equations and the assumption that Ohm's law may be used to relate the current at a point in the metal to the electric field at the same point. It is well known that Ohm's law must be generalized by introducing a complex conductivity when the frequency is so high that the period of the applied field becomes comparable with the time of relaxation of the electrons (Drude 1904, compare also Wilson 1936, p. 124). Relaxation effects of this nature, however, play no significant role in the anomalous skin effect except at infra-red frequencies; they are considered briefly in section 4.7.1, but may be disregarded for the present. The generalization of Ohm's law which concerns us here is of a different type, and is connected with the very long free path 
of the conduction electrons when the conductivity is high; when the free path is comparable with the skin depth $\delta$, it is not permissible to assume that an electron moves under the influence of a constant field between collisions, and the current at a point in the metal will depend on values of the electric field at other points.

Mathematically, the departure from Ohm's law is a consequence of the appearance of the usual term $\mathbf{v} \cdot \operatorname{grad}_{\mathbf{r}} f$ in the Boltzmann equation. The present problem differs from most of the previous ones, however, in that the electric field in the metal is not given a priori, but must itself be determined by the theory. When the relation between the current and an arbitrary field $E(z)$ has been found by solving the Boltzmann equation, it must be combined with Maxwell's equation (59) to give an equation from which $E(z)$ can be calculated; the surface impedance can then be obtained from the equation

$$
Z=-\frac{4 \pi i \omega}{c^{2}} \frac{E(0)}{E^{\prime}(0)}
$$

which follows directly from (59) and (63).

\subsection{The fundamental equation}

So long as we are only interested in the surface impedance of the metal (and not, for example, in the transmission coefficient of a thin film), the penetration depth of the field may always be treated as small compared with the linear dimensions of the specimen, and we may continue to consider a semi-infinite slab of metal. The Boltzmann equation then takes the same form (9) as in the discussion of the d.c. conductivity of a thin film, namely

$$
\frac{\partial f_{1}}{\partial z}+\frac{f_{1}}{\tau v_{z}}=\frac{\epsilon}{m v_{z}} \frac{\partial f_{0}}{\partial v_{x}} E(z),
$$

where the electric field is now, however, a function of $z$. (The influence of the highfrequency magnetic field may be neglected in writing down equation (66).) The general solution is now

$$
f_{1}(\mathbf{v}, z)=\exp \left(-\frac{z}{\tau v_{z}}\right)\left\{F(\mathbf{v})+\frac{\epsilon}{m v_{z}} \frac{\partial f_{0}}{\partial v_{x}} \int^{z} E(t) \exp \left(\frac{t}{\tau v_{z}}\right) \mathrm{d} t\right\} .
$$

The value of $F(\mathbf{v})$ when $v_{z}>0$ is determined in the usual way by the nature of the scattering at the surface of the metal, which is described by a reflection coefficient $p$; when $v_{z}<0, F(\mathbf{v})$ must be chosen so that $f_{1}$ does not become exponentially large as $z \rightarrow \infty$. Defining $E(-z)=E(z)$ for $z<0$, the solution which satisfies the boundary conditions may be written in the form

$$
\left.\begin{array}{rlr}
f_{1}^{+}(\mathbf{v}, z)= & \frac{\epsilon}{m v_{z}} \frac{\partial f_{0}}{\partial v_{x}} \exp \left(-\frac{z}{\tau v_{z}}\right)\left\{p \int_{-\infty}^{z} E(t) \exp \left(\frac{t}{\tau v_{z}}\right) \mathrm{d} t\right. & \\
& \left.+(1-p) \int_{0}^{z} E(t) \exp \left(\frac{t}{\tau v_{z}}\right) \mathrm{d} t\right\} & \left.\left(v_{z}>0\right),\right\} \\
f_{1}^{-}(\mathbf{v}, z)= & -\frac{\epsilon}{m v_{z}} \frac{\partial f_{0}}{\partial v_{x}} \exp \left(-\frac{z}{\tau v_{z}}\right) \int_{z}^{\infty} E(t) \exp \left(\frac{t}{\tau v_{z}}\right) \mathrm{d} t & \left(v_{z}<0\right) .
\end{array}\right\}
$$

The first of these expressions shows that the effect of partially elastic scattering may be described formally by replacing the semi-infinite metal slab by an infinite metal in 
which the field in the region $z<0$ is an image of the field in the region $z>0$ and in which a fraction $p$ of the electrons moves through the image field.

4.3.1. The calculation of the current density is now straightforward, and the result is

$$
J(z)=\frac{3 \sigma_{0}}{4 l}\left\{p \int_{-\infty}^{\infty} k\left(\frac{z-t}{l}\right) E(t) \mathrm{d} t+(1-p) \int_{0}^{\infty} k\left(\frac{z-t}{l}\right) E(t) \mathrm{d} t\right\}
$$

where

$$
k(u)=\int_{1}^{\infty}\left(\frac{1}{s}-\frac{1}{s^{3}}\right) e^{-s|u|} \mathrm{d} s
$$

it is seen that the current density at $z$ involves the values of the electric field at all points in the metal. The two integrals on the right-hand side of (69) represent the contributions to $J$ of the electrons which suffer specular and diffuse reflection respectively at the surface of the metal. We may note here that in the anomalous skin effect the value of $p$ plays only a subordinate part in determining the surface impedance (see section 4.6.1); there is thus a basic difference between the present phenomenon and the d.c. thin-conductor free-path effects which are in general entirely due to the presence of diffuse scattering at the boundaries.

4.3.2. The equation for $E$ is obtained by combining (59) and (69). It is convenient to introduce dimensionless coordinates $x=z / l, y=t / l$, and to write $E(l x)=f(x)$; the fundamental equation of the problem is then obtained in the form

$$
f^{\prime \prime}(x)=i \alpha\left\{p \int_{-\infty}^{\infty} k(x-y) f(y) \mathrm{d} y+(1-p) \int_{0}^{\infty} k(x-y) f(y) \mathrm{d} y\right\},
$$

where

$$
\alpha=3 \pi \omega l^{2} \sigma_{0} / c^{2}=\frac{3}{2} l^{2} / \delta^{2} .
$$

For the evaluation of the surface impedance it is only necessary to know $f(0) / f^{\prime}(0)$ (see equation (65)), and $f(x)$ is not required explicitly.

It is easily shown that the results of section 4.2 are regained in the limit where $\alpha \ll 1$.

\subsection{Solution of the fundamental equation}

In the two limiting cases $p=1$ and $p=0$ equation (71) can be solved by standard methods based on the theory of Fourier integrals (Titchmarsh 1937). When $p=1$, for example, the equation reduces to

$$
f^{\prime \prime}(x)=i \alpha \int_{-\infty}^{\infty} k(x-y) f(y) \mathrm{d} y,
$$

where $f(x)$ is, by definition, an even function of $x$. It is easy to see that $f^{\prime}(x)$ is discontinuous at $x=0$, and tends to a limit $\mu$ (say) as $x \rightarrow+0(-\mu$ as $x \rightarrow-0)$. The formal solution of (73) now proceeds as follows. Let

$$
\phi(t)=\int_{-\infty}^{\infty} f(x) e^{-i x t} \mathrm{~d} x=2 \int_{0}^{\infty} f(x) \cos x t \mathrm{~d} x
$$


and

$$
\kappa(t)=\int_{-\infty}^{\infty} k(x) e^{-i x t} \mathrm{~d} x .
$$

From (74), integrating by parts twice, it follows that

$$
\int_{-\infty}^{\infty} f^{\prime \prime}(x) e^{-i x t} \mathrm{~d} x=-t^{2} \phi(t)-2 \mu
$$

on the other hand, (73) implies that

$$
\begin{aligned}
\int_{-\infty}^{\infty} f^{\prime \prime}(x) e^{-i x t} \mathrm{~d} x & =i \alpha \int_{-\infty}^{\infty} e^{-i x t} \mathrm{~d} x \int_{-\infty}^{\infty} k(x-y) f(y) \mathrm{d} y \\
& =i \alpha \kappa(t) \phi(t)
\end{aligned}
$$

(inverting the order of integration). Hence, from (76) and (77),

$$
\phi(t)\left\{t^{2}+i \alpha \kappa(t)\right\}=-2 \mu,
$$

and the inversion formula for Fourier transforms gives the final result

$$
f(x)=-\frac{2 \mu}{\pi} \int_{0}^{\infty} \frac{\cos x t \mathrm{~d} t}{t^{2}+i \alpha \kappa(t)} .
$$

For $x=0$, in particular, it follows that

$$
\frac{f(0)}{f^{\prime}(0)}=-\frac{2}{\pi} \int_{0}^{\infty} \frac{\mathrm{d} t}{t^{2}+i \alpha \kappa(t)} .
$$

The explicit form of $\kappa(t)$ is readily obtained from (70) and (75), and is

$$
\kappa(t)=2 t^{-3}\left\{\left(1+t^{2}\right) \tan ^{-1} t-t\right\} .
$$

4.4.1. When $p=0$, equation (71) is of the same general type as the well-known integral equation of Milne which has been much studied in the theory of radiative transfer and in neutron diffusion problems. The solution in this case is based on the method which has been applied to Milne's equation by Wiener and Hopf; for details of the analysis the reader is referred to Reuter and Sondheimer's paper. The results are complicated but lead to conclusions which are generally similar to those found for $p=1$ (see section 4.6).

\subsection{The electric field}

The electric field $f(x)$ is not itself required for the evaluation of the surface impedance, but its behaviour is of interest. It is clear from (78) that $f(x)$ is not of exponential form, and in general its evaluation is complicated; for large values of $x$, however, it may be shown (Reuter and Sondheimer 1948, appendix III) that, when $p=1$,

$$
f(x)=C_{1} e^{-s_{1} x}+C_{2} e^{-x} / x^{2},
$$

where $C_{1}$ and $C_{2}$ are constants, and where $s_{1}$ is a complex parameter whose real part $\mathcal{R} s_{1}$ increases steadily with $\alpha$ and equals unity when $\alpha=\alpha_{0} \approx 2.63$.

The physical significance of this result is best understood by considering the case of a metal which is unbounded in all directions (Pippard, Reuter and Sondheimer 1948). In this case, when $\alpha \leqslant \alpha_{0}$, the electric field is found to be the simple damped 
exponential wave $e^{-s_{1} x}$, the penetration depth of which is $l / \mathcal{R} s_{1}$ and is thus equal to $l$ when $\alpha=\alpha_{0} \cdot \dagger$ For $\alpha>\alpha_{0}$ there are no solutions at all, which means that an exponential wave which is attenuated by a factor $e$ in a distance smaller than the free path cannot exist: it would correspond to a situation in which the contribution to the current at a point is greater the further the electrons contributing to the current have travelled in the wave direction, so that the expression for the resultant current density at any point diverges.

It is therefore clear that, in order to obtain solutions for all values of $\alpha$, it is essential to take the boundary conditions at the surface of the metal specifically into account in the calculation; it is to be expected, furthermore, that there will be a fundamental difference in the nature of the electric field for values of $\alpha$ less or greater than the critical value $\alpha_{0}$. This is in accordance with the form of equation (81). When $\alpha<\alpha_{0}$, the first term is dominant and the field in the metal, at sufficient distances from the surface, is unaffected by the presence of the surface, being similar in form to the solution for an infinite metal; when $\alpha>\alpha_{0}$, however, the second term is dominant, and the form of the excitation is now conditioned at all points by the presence of the surface and never approximates to a simple exponential form, being, in fact, a true surface excitation. In the latter case the electric field, though largely confined to the surface, has a long 'tail' of small amplitude which is effectively damped out in a distance of the order of $l$ and which may be regarded as being transmitted into the metal by electrons which move in the interior under the influence of no forces except their collisions with the lattice.

\subsection{The surface impedance}

The surface impedance can be obtained from (65) and (79) (or the corresponding expression for $p=0$ ), and in general the integrals have to be evaluated numerically. Explicit expressions for $Z$ can, however, be obtained in the limiting case $(\alpha \gg 1)$ when the free path is very large.

\subsubsection{The asymptotic expressions for $Z$}

The limiting value of $f(0) / f^{\prime}(0)$ when $\alpha \gg 1$ is obtained from (79) by replacing $\kappa(t)$ by its approximate value $\pi / t$ for large $t$. The integral is then readily evaluated and leads to the following limiting value of $Z$ when $p=1$ :

$$
Z_{\infty}=\frac{8}{9}\left(\sqrt{3 \pi \omega^{2} l / c^{4} \sigma_{0}}\right)^{1 / 3}(1+\sqrt{3 i}) .
$$

The corresponding result when $p=0$ is

$$
Z_{\infty}=\left(\sqrt{3 \pi \omega^{2} l / c^{4} \sigma_{0}}\right)^{1 / 3}(1+\sqrt{3 i})
$$

These expressions are to be contrasted with the result (64) which holds when $\alpha \ll 1$. We see that $Z$ is independent of $l$ when the free path is very large, in accordance with the experimental results; further, $Z$ varies with frequency as $\omega^{2 / 3}$, and the surface resistivity and reactance are related by

$$
X_{\infty}=\sqrt{3 R_{\infty}}
$$


under extreme anomalous conditions. These results hold both for $p=1$ and for $p=0$, and it is evident that although the precise nature of the surface scattering plays some part in determining the surface impedance, the effect is not considerable.

\subsubsection{The 'ineffectiveness concept'}

The physical principles underlying these results are not readily apparent from the detailed calculation, and it is therefore worth while to outline a simple qualitative theory which was developed by Pippard (1947) before the exact solution had been obtained. When the free path is large compared with the penetration depth, the electrons may be divided into two groups, those moving at small angles to the surface which have a reasonable chance of colliding in the surface layer, and the rest whose chance of such a collision is small. Pippard gave reasons for supposing that only the former group of electrons contributes effectively to the resistance, and the proportion of effective electrons may thus be written $\beta \delta^{\prime} / l$, where $\delta^{\prime}$ is the (unknown) effective penetration depth and where $\beta$ is a numerical constant of order unity. It is now supposed that the ineffective electrons may be entirely neglected, and that the effective electrons move in a constant field during a free path, so that the theory of the normal skin effect applies to them. The effective conductivity is then $\sigma^{\prime}=\beta \delta^{\prime} \sigma_{0} / l$, and $\delta^{\prime}$ is given by an expression of the form (62) with $\sigma_{0}$ replaced by $\sigma^{\prime}$. We thus obtain an implicit equation for $\delta^{\prime}$, which, when solved, gives

$$
\delta^{\prime}=\left(c^{2} l / 2 \pi \omega \beta \sigma_{0}\right)^{1 / 3} .
$$

Inserting $\sigma^{\prime}$ instead of $\sigma_{0}$ in (64) and using (85), we obtain the surface resistivity in the form

$$
R_{\infty}=\left(4 \pi^{2} \omega^{2} l / \beta c^{4} \sigma_{0}\right)^{1 / 3}
$$

in qualitative agreement with (82) and (83).

Later work on the interpretation of the theory (Marcus, to be published) has shown that the physical picture underlying the ineffectiveness concept must not be taken too literally, but it does provide a useful guiding principle which is of particular value when considering more complicated situations for which no exact theory exists (compare, for example, section 4.7.2).

4.6.3. Since $E(0) / E^{\prime}(0)=l f(0) / f^{\prime}(0)$, equations (65) and (79) show that $Z$ depends on the free path both through $l$ and $\alpha$. It is convenient to express $Z$ as a function only of $\alpha$ and of quantities independent of $l$; using equations (1b) and (72) to eliminate $l$, we find that

$$
Z=-i \sqrt{\frac{8}{3}} A \alpha^{1 / 3} f(0) / f^{\prime}(0),
$$

where

$$
A=\sqrt{6}\left(\frac{\pi \omega}{\epsilon c^{2}}\right)^{2 / 3}\left(\frac{m \bar{v}}{3 n}\right)^{1 / 3}
$$

$A$ is constant for a given metal and a given frequency and has been chosen such that, when $\alpha \ll 1$,

$$
Z=A \alpha^{-1 / 6}(1+i)
$$




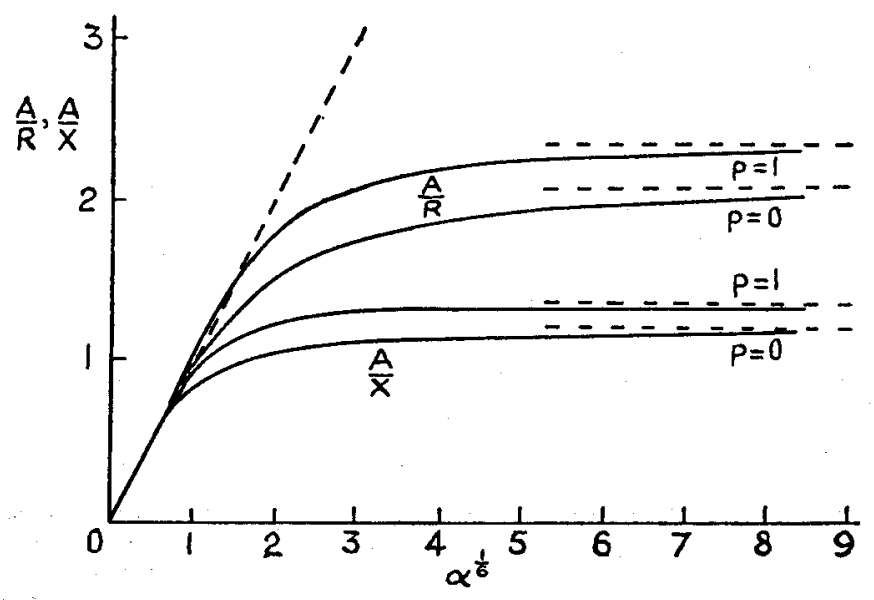

Figure 10. The theoretical high-frequency surface impedance of metals at low temperatures.

as is easily verified. When $\alpha \gg 1$, we obtain

$$
Z_{\infty}=\frac{4 \sqrt{2}}{9 \pi^{1 / 3}} A(1+\sqrt{3 i} \quad(p=1)
$$

and

$$
Z_{\infty}=\frac{A}{\sqrt{2 \pi^{1 / 3}}}(1+\sqrt{3 i} \quad(p=0) ;
$$

equations (89), (90), and (91) are of course equivalent to (64), (82), and (83). The complete theoretical curves are shown in figure 10, where $A / R$ and $A / X$ have been plotted against $\alpha^{1 / 6}$.

\subsubsection{Comparison with experiment}

The experimental method used by Pippard to measure $R$ involves the construction of a resonator of the metal to be studied and the determination of its selectivity $Q$; for a given resonator, $Q$ is proportional to the surface conductivity $1 / R$. The results for any metal are exhibited by plotting $1 / R$ against $\sqrt{ } \sigma_{0}$ at constant frequency. Since $\sqrt{ } \sigma_{0}$ and $\alpha^{1 / 6}$ both vary as $\sqrt{ } l$, the experimental points can be scaled directly to fit the theoretical curve if it is assumed that $p$ equals either 1 or 0 . It is then possible, by reading off the value of $\alpha$ corresponding to any pair of values of $R$ and $\sigma_{0}$, to estimate $\sigma_{0} / l$ for any metal; according to equations (62) and (72), $\sigma_{0} / l=\sqrt{ }\left(3 \pi \omega \sigma_{0}^{3} / c^{2} \alpha\right)$.

Pippard's (1947) results were in qualitative agreement with the theory, but were not sufficiently detailed to indicate which value is to be chosen for $p$. Chambers (1950b) has recently carried out more extensive experiments, in which the temperature was varied between $2^{\circ} \mathrm{K}$ and $90^{\circ} \mathrm{K}$; the frequency (as in Pippard's experiments) was $1200 \mathrm{Mc} / \mathrm{s}$, corresponding to a free-space wavelength of $25 \mathrm{~cm}$. Some of Chambers results for copper, silver, and tin are shown in figure 11, scaled (I) to fit the theoretical curve to $p=0$, and (II) in an attempt to fit the curve for $p=1$. The agreement with curve I is really excellent, and it thus appears that the reflection at the surface is completely diffuse at all temperatures for all specimens. 


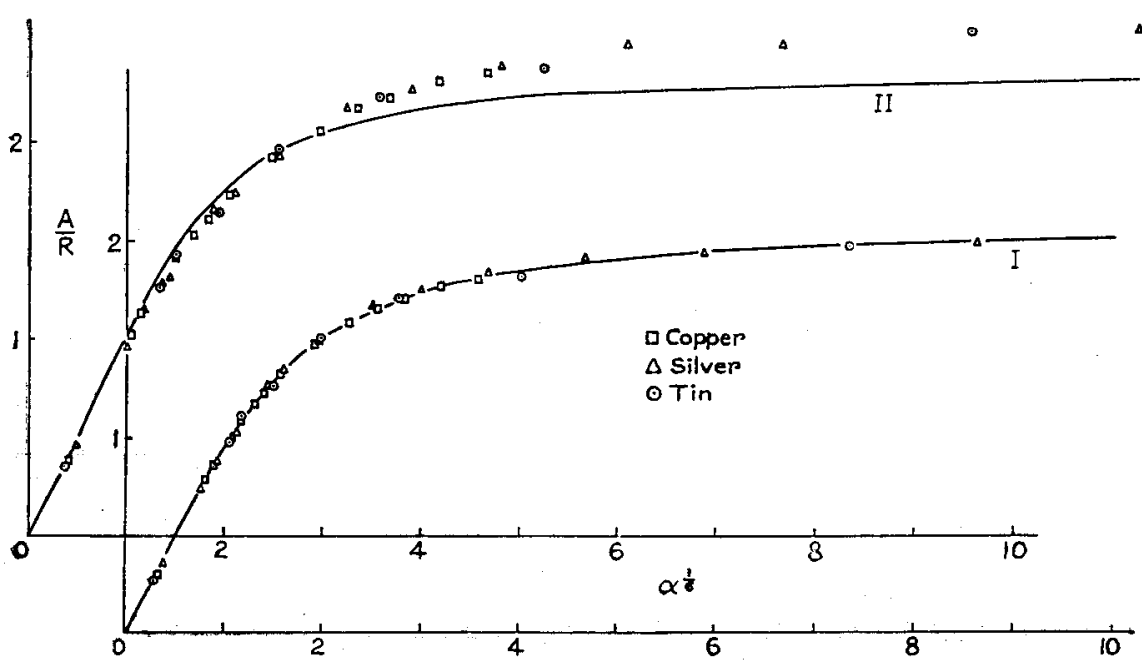

Figure 11. Experimental results on the surface resistance at low temperatures. (I) Experimental points fitted to $p=0$ curve. (II) Attempt to fit to $p=1$ curve.

4.6.4.1. These experiments, as Chambers has pointed out, suggest strongly that the value of $p$ which describes the situation in real metals is zero in all cases, including the d.c. size effects (compare sections 2.4.1 and 2.4.1.1). It should be noted that the appropriate value of $p$ need not necessarily be the same for all the different problems studied: although $p$ has been defined in the same way in each case, it probably represents some kind of average value of some more complicated quantity, and there is no reason to suppose that this average should have the same value in all cases. If Pippard's ineffectiveness concept is taken literally, the value of $p$ in the anomalous skin effect is determined by the behaviour on reflection of electrons travelling at very small angles to the surface. If specular reflection occurs at all, it seems most likely to occur for these electrons, and since it is not observed for them (i.e. since $p=0$ in the anomalous skin effect), it is reasonable to expect that $p=0$ also for the d.c. case, where electrons travelling at all angles to the surface are 'effective'.

4.6.4.2. Values of $\sigma_{0} / l$ deduced from the experiments are shown in table 3 , together with the corresponding values of $n / n_{a}$, the number of conduction electrons

Table 3. Estimates of $\sigma_{0} / l$ and $n / n_{a}$ from observations on the anomalous skin effect.

\begin{tabular}{lcl}
\hline Metal & $\begin{array}{c}\left(\sigma_{0} / l\right) \times 10^{-22} \\
(\text { gaussian units })\end{array}$ & $n / n_{a}$ \\
\hline $\mathrm{Cu}$ & 13.9 & 1.0 \\
$\mathrm{Ag}$ & 8.3 & 0.68 \\
$\mathrm{Au}$ & 7.6 & 0.60 \\
$\mathrm{Sn}$ & 8.6 & 1.1 \\
$\mathrm{Hg}$ & 3.3 & 0.23 \\
$\mathrm{Al}$ & 5.5 & 0.37 \\
\hline
\end{tabular}


per atom. The values for copper, silver, gold, and tin were obtained by Chambers, $\uparrow$ and are subject to probable errors of about $\pm 5 \%$; the values for mercury and aluminium have been deduced from Pippard's measurements and have larger probable errors.

The results for the monovalent metals are in fair agreement with the expected value of one conduction electron per atom, although the figures for silver and gold are somewhat too low. The figures for tin and mercury may be compared with the corresonding figures $\left(n / n_{a}=0.43\right.$ and 0.15$)$ obtained from the thin-conductor experiments; the agreement is probably as good as can be expected for such complicated metals.

4.6.4.3. The present method of estimating the free path is free from the main difficulties which beset the thin-conductor experiments, such as the impossibility of measuring the bulk conductivity directly on the specimen, or the disturbing influence of the bulk magneto-resistance effect. It is, however, very important to ensure that the specimens have no surface layer with properties different from those of the bulk metal: if, for example, there is a thin surface layer, of thickness comparable with the skin depth, with an abnormally high residual resistance, the temperature variation of the surface resistivity will simulate the behaviour in the anomalous skin effect, although in this case the high resistivity will not be a result of the long free path. This is in fact what appears to have happened in early measurements by Pippard on wires of the noble metals, which led to very low values of $n / n_{a}$. The process of drawing wires of these metals produces an amorphous layer which is not easily removed by annealing, but which, in Chambers' experiments, was removed by electrolytic polishing. In subsidiary experiments Chambers has confirmed that the predominant cause of abnormally high surface losses is the presence of a high-resistance layer, and that possible alternative causes such as the roughness of the surface are generally of smaller importance.

Similar disturbing influences appear to have played a part in experiments on the anomalous skin effect carried out in the United States (Fairbank 1949, Maxwell, Marcus, and Slater 1949).

4.6.4.4. Pippard (1950a) has recently made experiments on tin using a frequency of $9400 \mathrm{Mc} / \mathrm{s}$, corresponding to a wavelength of $3.2 \mathrm{~cm}$. By comparing the results with the $25 \mathrm{~cm}$ measurements, Pippard confirmed that the asymptotic value of the surface resistance varies with frequency as $\omega^{2 / 3}$ for a given material; this law is found to be accurately obeyed. At the same time Pippard was able to estimate the surface reactance $X$ by an indirect method involving the superconducting behaviour of the metal. He found that $X_{\infty} / R_{\infty} \approx 1.76$ for tin, in quite as good agreement with the theoretical value $\sqrt{ } 3$ as may be expected. These experiments are considered further in section 4.72 .

\subsection{Extensions of the theory}

We conclude by considering briefly various generalizations of the basic theory given above.

$\uparrow$ These values differ from those given in the published note (Chambers 1950b); they are based on more accurate values of the d.c. conductivity $\sigma_{0}$. 


\subsubsection{Relaxation effects}

Up to now it has been assumed that the product $\omega \tau$ is small compared with unity. In the $9400 \mathrm{Mc} / \mathrm{s}$ experiments this condition is no longer satisfied, but we have seen that the results are in good agreement with the non-relaxation theory. This is explained by the extension of the theory to take account of relaxation, which may be obtained by replacing the time of relaxation in the Boltzmann equation (66) by a complex time of relaxation $\tau /(1+i \omega \tau)$. It is then found (Reuter and Sondheimer 1948 , section 6) that the integrals involve, instead of $i \alpha$, the generalized parameter $\xi=i \alpha /(1+i \omega \tau)^{3}$; so long as $|\xi| \gg 1$, however, the final expressions obtained for $Z$ are identical with (82) and (83), so that relaxation effects are negligible. The criterion $|\xi| \gg 1$ for neglecting relaxation is equivalent to the condition, which may be easily understood physically, that the period $1 / \omega$ of the applied field shall be large compared with, not $\tau$, but the time taken by an electron to traverse the skin depth, which is very much smaller than $\tau$. As a result it is not to be expected that relaxation effects will play any part at wavelengths greater than about $\frac{1}{10} \mathrm{~mm}$.

So long as the displacement current is negligible, the absorption coefficient $\mathcal{A}$ of a metal for normally incident radiation, defined as the ratio of the intensities of the absorbed and incident waves, is directly proportional to the surface resistivity. According to the usual relaxation theory $\mathcal{A}$ varies as $\sqrt{ } \omega$ at low frequencies (this is the Hagen-Rubens relation), but is independent of frequency when $\omega \tau \gg 1$. The difference in relaxation behaviour which the anomalous skin effect introduces at low temperatures is illustrated in figure 12, which shows the theoretical absorption coefficient of a good silver specimen at liquid-helium temperatures and at frequencies ranging from long radio waves to ultra-violet light. The anomalous effects are most marked in the long infra-red, while at higher frequencies the relaxation effects tend to restore the normal behaviour; the maximum value of $\mathcal{A}$ in figure 12 is $1.5 \times 10^{-3}$ and occurs at a wavelength of $70 \mu$.

The absorptivity of pure electropolished metals for infra-red radiation of wavelength $14 \mu$ has recently been measured by Ramanathan (unpublished) at liquid-helium temperatures. The observed behaviour is not in agreement with the

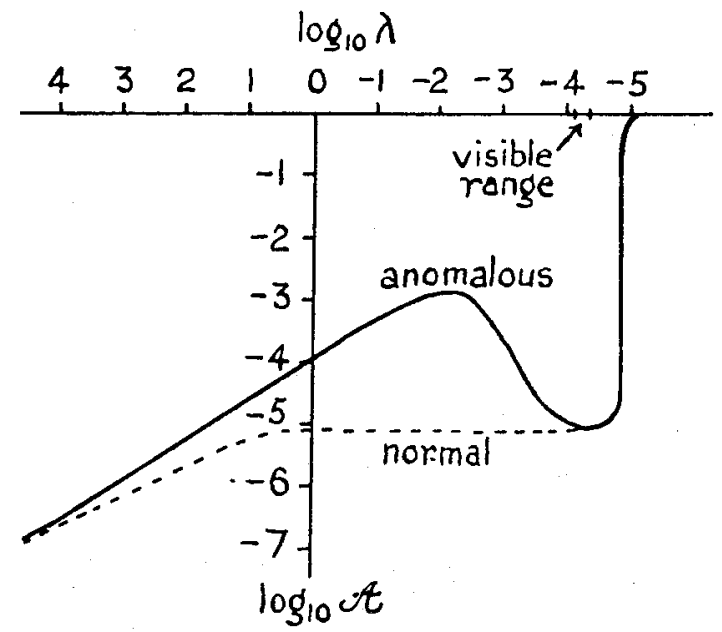

Figure 12. Theoretical absorption coefficient of silver at liquid-helium temperatures for $p=1$, plotted logarithmically against the wavelength $\lambda$. 
theory: the absorptivity in all cases exceeds the theoretical value by a factor of the order of 10, and is in fact about as great as the value obtained by extrapolating the $\omega^{2 / 3}$ law which holds when relaxation effects are negligible. The origin of the discrepancy is not clear at present, but it is possible that the disagreement with the relaxation theory is only apparent and is connected with the value of the surface reflection coefficient $p$. $p$ was assumed to be unity by Reuter and Sondheimer when evaluating their formulae for high frequencies; it has been reported that in the infrared region the absorptivity for $p=0$ is much higher than for $p=1$ (Holstein, unpublished calculations), but details of the computations are not yet available.

\subsubsection{The anomalous skin effect in anisotropic metals}

In his experiments at $9400 \mathrm{Mc} / \mathrm{s}$, Pippard (1950a) studied the surface resistance of single crystals of tin as a function of orientation. In figure $131 / R$, measured under extreme anomalous conditions, is displayed as a function of $\theta$, the angle between the tetrad axis of the crystal and the axis of the (cylindical) specimen, which is also the direction of current flow.

The form of the anisotropy of $1 / R$ is interesting, and differs from the result to be expected for a metal obeying Ohm's law. In an anisotropic metal the usual form of Ohm's law, $\mathbf{J}=\sigma \mathbf{E}$, must be generalized by treating $\sigma$ as a symmetric second-order tensor. The theory of the normal skin effect in such a metal has been considered by Pippard, who has shown that, for the conditions of his experiments, it leads to a monotonic variation of $R$ with $\theta$, which is at variance with the experimental results. Pippard has pointed out that the reason for the anomalous behaviour is to be found in the form of the equations connecting $\mathbf{J}$ and $\mathbf{E}$ in the normal and anomalous theories. In the former the relation is a linear point-relation whether the metal is isotropic or not, the value of $\mathbf{J}$ at any point being determined solely by the value of $\mathbf{E}$ at that point. In the anomalous theory, on the other hand, the relation between $\mathbf{J}$ and $\mathbf{E}$, while still linear, is no longer a point relation; there is then no longer any quantity analogous to $\sigma$ which may be simply generalized by the introduction of a tensor, and as a consequence the mathematical formulation of the problem permits a much greater freedom of behaviour in an anisotropic metal than is allowed by a pointrelation. It is clear, furthermore, that the free-electron model which we have always employed hitherto will be entirely inadequate to deal with the present problem, and the anisotropic behaviour will depend on the anisotropic form of the energy surfaces

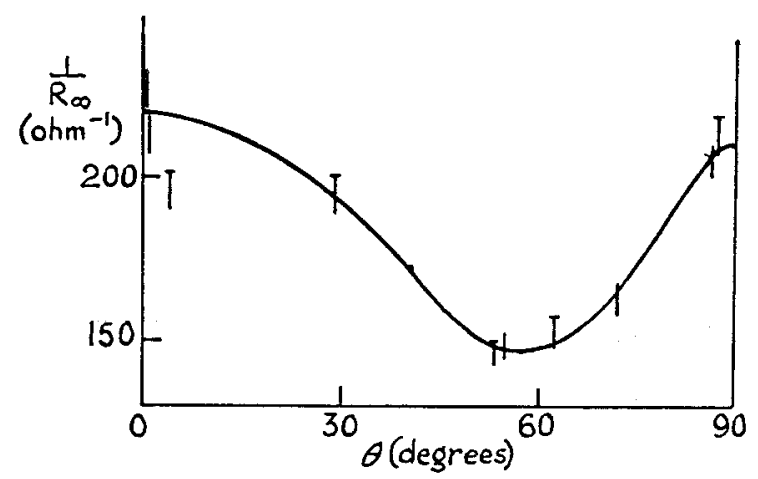

Figure 13. Variation with orientation of surface conductivity of single crystals of tin. 
in real metals. A first discussion of the problem using the ineffectiveness concept has been given by Pippard, but further investigation, both experimental and theoretical, is required before the phenomenon can be said to be fully understood.

\subsubsection{Application to superconductivity}

Most of the recent investigations on the high-frequency behaviour of metals at low temperatures have been concerned primarily with the properties of the superconducting state, a topic which we have ignored hitherto and which is outside the scope of the present article. It should be mentioned, however, that the methods used in the theory of anomalous skin effect find an application in the theoretical analysis of certain models of a superconductor, the so-called two-fluid models. The essential feature of these models is that the electrons in a superconductor may be thought of as belonging to either of two groups, superconducting and normal respectively, and that the current density may be written as the sum of separate contributions from each group, the supercurrent and the normal current. Under the influence of an electric field the normal electrons in a superconductor are supposed to behave in the same way as the electrons in a normal metal, and they will therefore exhibit similar free-path phenomena at high frequencies. The theory has been worked out in detail by Maxwell, Marcus and Slater (1949) for the particular model of a superconductor which is due to H. London (1940), and a careful critical discussion of the whole subject has been given by Pippard (1950b), whose paper should be consulted for further details. It must be emphasized that, since there is as yet no accepted and fully developed theory of superconductivity, the status of these calculations on superconductors is much more speculative than that of the theory which applies to normal metals.

\subsubsection{Magnetic effects}

We wish to point out, finally, that effects analogous to those considered in section 3 are to be expected at high frequencies if a steady magnetic field is superimposed on the specimen. In particular, if the magnetic field is perpendicular to the surface of the metal, the surface impedance will vary with $H$ in a manner which may be analysed theoretically by combining the methods of section 3.4 with those of the present section; at the same time there will be a high-frequency Hall electric field, and at low temperatures there will be an 'anomalous Hall effect'. The analysis of these phenomena, though laborious, is straightforward in principle, but elaborate calculations are probably not worth undertaking until expeimental data have become available.

\section{Acknowledgement}

My best thanks are due to Mr R. G. Chambers for several instructive discussions and for reading and commenting on the article in manuscript.

\section{References}

Andrew, E. R., 1949, Proc. Phys. Soc. A, 62, 77.

Chambers, R. G., 1950a, Proc. Roy. Soc. A, 202, 378; 1950b, Nature, Lond., 165, 239; 1951, PhD Dissertation, Cambridge.

Dingle, R. B., 1950, Proc. Roy. Soc. A, 201, 545.

Drude, P., 1904, Ann. Phys., 14, 936. 
Eucken, A., and Förster, F., 1934, Nach. Ges. Wiss. Göttingen, 1, 43, 129. See also papers by various authors in Ann. Phys., 1937-41.

Fairbank, W. M., 1949, Phys. Rev., 76, 1106.

Fuchs, K., 1938, Proc. Camb. Phil. Soc., 34, 100.

London, H., 1940, Proc. Roy. Soc. A, 176, 522.

Lovell, A. C. B., 1936, Proc. Roy. Soc. A, 157, 311.

MacDonald, D. K. C., I949, Nature, Lond., 163, 637; 1950, Proc. Phys. Soc. A, 63, 290.

MacDonald, D. K. C., and Sarginson, K., 1950, Proc. Roy. Soc. A, 203, 223.

Maxwell, E., Marcus, P. M., and Slater, J. C., 1949, Phys. Rev., 76, 1332.

Mott, N. F., and Jones, H., 1936, The Theory of the Properties of Metals and Alloys (Oxford: University Press), p. 268.

Nordheim, L., 1934, Act. Sci. et Ind., No. 131 (Paris: Hermann).

Peierls, R., 1934, Z. Phys., 88, 786; Helv. Phys. Acta, 7 (Supp.), 24.

Pippard, A. B., 1947, Proc. Roy. Soc. A, 191, 385; 1950a, Ibid., 203, 98; 1950b, Ibid., 203, 195.

Pippard, A. B., Reuter, G. E. H., and Sondheimer, E. H., 1948, Phys. Rev., 73, 920.

Reuter, G. E. H., and Sondheimer, E. H., 1948, Proc. Roy. Soc. A, 195, 336.

Sondheimer, E. H., 1950, Phys. Rev., 80, 401.

Thomson, J. J., 1901, Proc. Camb. Phil. Soc., 11, 120.

Titchmarsh, E. C., 1937, Introduction to the Theory of Fourier Integrals (Oxford: Clarendon Press), chapter 11.

Wilson, A. H., 1936, The Theory of Metals (Cambridge: University Press). 